\title{
Characterisation of an inhomogeneously irradiated microstrip detector using a fine spot infrared laser
}

\author{
G. Casse, S. F. Biagi, T.J.V. Bowcock, A. Greenall, J.P. Phillips, P.R. Turner and \\ V. Wright \\ Oliver Lodge Laboratory, University of Liverpool, P.O. Box 147, Liverpool L69 3BX, UK
}

\begin{abstract}
A prototype silicon microstrip detector for the $\mathrm{LHCb}$ vertex locator (VELO) has been partially irradiated using a $24 \mathrm{GeV} / \mathrm{c}$ proton beam at the CERN-PS accelerator. The detector possesses a radial strip geometry designed to measure the azimuthal coordinate (Phi) of tracks within the VELO. The peak fluence received by the detector was measured to be $4.6 \times 10^{14} \mathrm{p} / \mathrm{cm}^{2}$ though the non-uniform nature of the exposure left part of the detector unirradiated. The inhomogeneous irradiation introduced a damage profile in the detector approximating to that expected in the VELO. High irradiation gradients are important to study as they can modify the electric field within the silicon. Of special interest are changes in the component of the electric field parallel to the strip plane but perpendicular to the strips which lead to systematic shifts in the reconstructed cluster position. If these (flux and position dependent) shifts are sufficiently large they could contribute to a degraded spatial resolution of the detector. In order to quantify these effects a precise fine light spot infrared laser was used to investigate the charge collection properties of the sensor. Particular attention was devoted to the regions where a high gradient of the fluence introduced a large gradient in the effective local space charge. The results reported below place limits on the "distortions" expected in the VELO due to non-uniform irradiation.
\end{abstract}

\section{Introduction}

The silicon detectors for the VELO tracker [1] are designed to provide the azimuthal (phi measuring sensor) and radial (R measuring sensor) coordinates [2]. An $\mathrm{LHCb}$ Phi measuring detector was non-uniformly irradiated, with $24 \mathrm{GeV} / \mathrm{c}$ protons in the CERN-PS/T7 experimental area, to study the effects of an inhomogeneous damage profile in the detector bulk.

To study the position dependent properties (e.g. the depletion voltage and charge collection) of the irradiated sensor a precise method of injecting electron-hole

pairs is necessary. An infrared laser system has been built that allows the sensor to be scanned using a fine beam. The results of the study on a partially irradiated phi-type detector are presented below.

\section{LHCb prototype phi detector}

The LHCb prototype phi measuring sensors are $300 \mu \mathrm{m}$ thick, p-strip in n-bulk silicon, with a semicircular shape covering 182 degrees. The sensor masks were designed at the University of Liverpool and fabricated by Micron Semiconductor [3] 
using an oxygen enriched (density $\sim 2 \times 10^{17} \mathrm{~cm}^{-3}$ ) [8] FZ silicon 6" wafer. The sensors are divided into inner and outer radial sections each containing 1024 strips (Fig. 1). The inner strip and outer strips are collinear and are AC coupled to the p-implants on a "first" metal layer. The outer strips have bond-pads at the outer radius of the sensor. The inner strips are routed to the bonding pads, also located at the outer radius of the sensor, via metal strips. These metal routing strips are fabricated on a "second" metal layer and are insulated from the first metal layer by a 3.7-4 $\mu \mathrm{m}$ thick oxide layer. The routing lines from the inner section run in between the strips in the outer section. Some dimensions of the detector geometry are given below.

- The bonding pads have a pitch of $62 \mu \mathrm{m}$. To enable easier bond the pads for the inner strips are interleaved and staggered with those for the outer ones so that the effective pitch is $124 \mu \mathrm{m}$.

- The pitch of the innermost end of an outer strip is $\sim 55.5 \mu \mathrm{m}$.

- The pitch of the innermost and outermost ends of an inner strip are $\sim 24.4 \mu \mathrm{m}$ and $\sim 55.4 \mu \mathrm{m}$ respectively.

- The strip width increases with the radius. The width of the innermost end of an inner strip is $\sim 12 \mu \mathrm{m}$ and the outermost end is $\sim 16.7 \mu \mathrm{m}$. The width of the innermost end of an outer strip is $\sim 16.8 \mu \mathrm{m}$ and the outermost end is $\sim 37 \mu \mathrm{m}$.

- The detector is also divided in 8 sectors, each one with bonding pads for the 128 inner and 128 outer strips.

\section{Inhomogeneous irradiation of the Phi detector}

A schematic of the set-up used to irradiate the Phi detector with $24 \mathrm{GeV} / \mathrm{c}$ protons in the CERN-PS East Hall is shown in Fig. 2. A holder containing the detector was mounted in a motorised carrier (Shuttle) in the IRRAD-1 facility [4]. The Shuttle allows positioning of the detector in the beam with the beam centre aligned as shown in Fig. 2. The beam has a nearly Gaussian profile with a FWHM of approximately $2 \mathrm{~cm}$. Fig. 3 shows the fluence profile as measured using the activated aluminium foil technique [5] during the irradiation. The error on the absolute magnitude of the fluence is about $10 \%$. The sensor position relative to the beam centre is known to an accuracy of about $2 \mathrm{~mm}$. The radiation damage introduces changes in the detector reverse current and effective space charge $\left(N_{\text {eff }}\right)$. The reverse current and, for high doses, $N_{\text {eff }}$ increase proportionally to the fluence. As a consequence of the beam profile, the detector $N_{\text {eff }}$ has a nearly Gaussian profile across the device.

The detector was irradiated during $\sim 14$ hours between the $7^{\text {th }}$ and the $8^{\text {th }}$ of October 2000. The temperature of the irradiation area was about $32^{\circ} \mathrm{C}$. After irradiation the detector was kept at $\sim-20^{\circ} \mathrm{C}$ to freeze the annealing process, until it was sent to Liverpool for measurement. During transport $(\sim 48 \mathrm{~h})$ the temperature was not monitored, but it is safe to assume that it never exceeded $20^{\circ} \mathrm{C}$. On arrival at Liverpool the detector was stored at $\sim-25^{\circ} \mathrm{C}$. The detector was removed from cold storage and glued onto a support (necessary for bonding) and kept in a room at $25^{\circ} \mathrm{C}$ for 12 hours to allow curing of the glue. The equivalent annealing time corresponds to 5 days at room temperature $\left(20^{\circ} \mathrm{C}\right.$ in Oct $)$. 


\section{Experimental methods}

\section{a. The detector support}

To study the various sectors of the detector using a single read-out chip with 128 channels, a dedicated rebondable support, an infrared laser system and a precise $\mathrm{x}-\mathrm{y}$ table have been set-up. The support (Fig. 4) has been designed to allow the sensor to be rotated hence enabling each sector to be bonded in turn. A rebondable fan-in extension was added to permit multiple bonding to the chip fan-in.

A single chip with 128 channels was used to read-out the outer radial section. In order to study the effect of the routing lines (from the inner radial section) on the electrical properties of the detector, two sets of read-out channels (generally 10) at both sides of each sector studied, were bonded to the outer strips and the intermediate routing lines alternatively. All the remaining channels were bonded to the outer strips only, leaving the routing lines floating.

\section{b. The read-out system}

The sensor was read-out by the SCT128 LHC speed electronics [7]. The output of the chip is a data stream of 128 channels divided into $25 \mathrm{~ns}$ time bins containing single channel information. This output was acquired by the LeCroy LC574AL $1 \mathrm{GHz}$ oscilloscope and averaged over 1000 sweeps to record the height of the signal. This technique strongly suppresses the effect of electronic noise.

The read out speed was $40 \mathrm{MHz}$ and the peaking time about $25 \mathrm{~ns}$. The timing of the readout relative to the laser pulse was adjusted to optimise the signal size. The signal was recorded for different trigger delays time allowing the shape of the signal to be deduced. This shape is compared to the design pulse shape in Fig. 5.

An asymmetry due to the read-out system was found. The time "bin" that follows the hit channel exhibits a negative signal, shown in Fig. 6, where the pulse is injected every four channels using the internal calibration of the SCT128 chip. Two heights of calibration pulse were injected and, in both cases, a large (10-15\%) negative signal was observed in the adjacent time bin. A correction for this effect has been applied.

\section{c. Infrared laser set-up and mechanics}

An infrared diode laser with $1060 \mathrm{~nm}$ wavelength was driven by an external pulse generator to deliver short $(<5$ ns FWHM, Fig. 7) pulses. The light was guided by a single mode optical fibre to a light splitter with three output lines delivering about $60 \%, 20 \%$ and $20 \%$ of the input intensity respectively. The $60 \%$ output was connected via a single mode optical fibre and a coupler to a TTI TIA-950 Optical Electrical converter connected to the oscilloscope. This was used to monitor the stability of the laser power emission. A $6 \mu \mathrm{m}$ diameter core fibre, terminated with an optical focuser of $12 \mathrm{~mm}$ focal length, was connected to one of the $20 \%$ output lines. The light beam had a slightly elliptical profile with full-width-half-maxima of $7 \mu \mathrm{m}$ and $6.6 \mu \mathrm{m}$, and widths of $13.9 \mu \mathrm{m}$ and $12.9 \mu \mathrm{m}$ at $13 \%$ of the maximum peak intensity on the major and minor axes. The laser power output was adjusted to produce a signal corresponding to 3 minimum ionising particles in the sensor.

The sensor was mounted on an x-y table perpendicular to the beam (Fig. 8). Two micromanipulators allowed precise movement with a resolution of two microns. 


\section{Experimental results}

\section{a. Full depletion voltage $\left(V_{f d}\right)$ profile}

The charge collection efficiency (CCE) allows the extraction of the local $V_{f d}$ [9]. $V_{f d}$ is proportional to $N_{\text {eff }}$

$$
V_{f d}=q_{0} w^{2} N_{e f f} / 2 \varepsilon_{S i}
$$

where $q_{0}$ is the electron charge, $w$ is the thickness of the detector and $\varepsilon_{S i}$ is the dielectric constant of silicon.

The collected (cluster) charge was defined to be the sum of the charge collected on a strip and the two neighbouring strips (and routing lines where they were connected) on each side. An example of CCE as a function of bias voltage for a low irradiation region $\left(<5 \times 10^{13} \mathrm{p} / \mathrm{cm}^{2}\right)$ of the sensor is shown in Fig 9a. The CCE curves are normalised to the maximum charge collected with strong over-depletion $(500 \mathrm{~V})$. The collected charge can be seen to rise approximately linearly at both very low and high voltages, with a smooth transition between these two regions of linear behaviour. At low voltages the charge collected rises rapidly, as the depleted depth increases, and at high voltages the sensor is in a plateau region corresponding to full depletion. The two linear regions were fitted with straight lines, which were extrapolated into the transition region. The intersection of the lines characterizes the centre of the transition from one behaviour to the other and this was taken as an estimate of $V_{f d}$.

The low irradiation region (Figure 9(a)) may be compared a region that has received a high dose (Figure $9(\mathrm{~b})$ ) (about $4.4 \times 10^{14} \mathrm{p} / \mathrm{cm}^{2}$ ). It can be seen that irradiated region has a higher $V_{f d}$ and that the plateau region still exhibits a significant rising trend. In this case the maximum bias limited the number of measurement in the plateau region. This led to a larger uncertainty in the evaluation of $V_{f d}$. The error on $V_{f d}$ was estimated using a different choice of points for the two trend lines. The CCE is arbitrarily normalized at the maximum of the CCE-voltage curve. Further studies are being performed to establish the absolute amount of charge collected at a specific bias voltage.

Fig. 10 shows an example of charge collection efficiency (CCE) curves in three different positions and estimated radiation fluences $\left(<5 \times 10^{13}, 1.2 \times 10^{14}, 3.4 \times 10^{14}\right.$ $\left.\mathrm{p} / \mathrm{cm}^{2}\right)$ of the detector. Fig. 11 shows the map of $V_{f d}$ measured across the whole detector at the outer end of the outer radial section. The shape of $V_{f d}$ closely follows the radiation damage profile. Between strip \#570 and \#670 the gradient of $V_{f d}$ (and therefore of $\left.N_{\text {eff }}\right)$ is positive, while between strip \#690 and \#760 it is negative. Transverse component of the electric field generated by the inhomogeneity of the irradiation would thus be expected to be opposite direction in these two regions. The comparison of the CCE properties in these two regions enables the study of the effects of a possible transverse electric field.

\section{b. Noise}

In the low depletion voltage region (low fluence), noise due to micro-discharges [10] may be observed. In the more irradiated area the bias could be raised above 400 volts without any increase in the noise. Fig. 12 shows the noise measured in outer radial sector 5 of the sensor (Fig. 1) as a function of voltage. The noise is not flat 
across the chip because of the poor condition of the fan-in extension. This measurement was not intended to determine the absolute noise level of the detector, but to give evidence of micro-discharges above 200Volts in the low fluence area $\left(<1.0 \times 10^{14}\right)$. With the same applied bias voltage, the electric field is much higher in the low $V_{f d}$ region than in the high one, explaining the shape of the measured noise.

In Fig. 12(c) and (d) the noise values for the strips between \#549 and \#559 (in the low fluence region) are missing because of an automatic masking, within the analysis program, of channels with extreme noise.

\section{c. Charge sharing between adjacent strips}

A detailed study of the charge division between adjacent strips (called Left and Right for simplicity) has been carried out in different areas of the detector. The charge division has been evaluated using the pulse height as a function of the local coordinate $x$, defined as the distance in microns of the centre of the light spot from the centre of the left (L) strip. The following algorithm has been used to evaluate the ratio $(\eta)$ of the charge seen by the right strip $(\mathrm{R})$ to the total charge:

$$
\eta=H_{R} /\left(H_{R}+H_{L}\right)
$$

where $H_{R}$ and $H_{L}$ are the height of the signals seen by the left and right strip respectively.

The read-out system introduces a distortion in the time bin that follows the signal bin, as described above. Fig. 13 shows the charge sharing as a function of $x$ for the two different bonding schemes. In Fig. 13a two adjacent strips were bonded to adjacent read out channels so that the signal of the right strip is in the time bin that follows the left strip signal. In this case an asymmetry is found in the $\eta$ function: no charge is seen on the R strip at the local coordinates 30 and $40 \mu \mathrm{m}$. This is because the over-shoot compensates the small charge picked by strip R In Fig. 13b the intermediate routing line is also bonded to the read out chip, in the time bin that follows the signal on the left strip. In this second case the over-shoot does not affect the signal in the right strip, which is two time bins away from the strip signal. The over-shoot affects the routing line and does not disturb the charge seen by strip R. In this second case the symmetry of the charge-sharing scan across two strips is very good. This allows the application of a correction for the $\eta$ scan performed between strips measured leaving the intermediate routing line floating.

Figures from 14 to 19 show the $\eta$ scan as measured in the outer end of the outer radial section.

Fig. 14 shows the $\eta$ scan for a non-irradiated area (strips \#244-245) $(<5 \times$ $\left.10^{13} \mathrm{p} / \mathrm{cm}^{2}\right)$. Fig. 15 is taken from strips located in the area where the gradient of $V_{\mathrm{fd}}$ is small (\#490-491) $\left(<5 \times 10^{13} \mathrm{p} / \mathrm{cm}^{2}\right)$ and the depletion voltage is about 50 Volts. Fig. 16 is from an area where the gradient of $V_{f d}$ is increasing from strip L towards strip $\mathrm{R}(\# 634-635)\left(3.9 \times 10^{14} \mathrm{p} / \mathrm{cm}^{2}\right)$. Fig. 17 is from strips located in the area where the gradient of $V_{f d}$ is small but with a high radiation level (\#690-691) $\left(4.4 \times 10^{14} \mathrm{p} / \mathrm{cm}^{2}\right)$. Fig. 18 shows the area where the gradient is decreasing from strip L towards strip R $(\# 760-761))\left(2.2 \times 10^{14} \mathrm{p} / \mathrm{cm}^{2}\right)$. There is no evidence of distortion due to a transverse electric field in the region with maximum gradient of $V_{f d}$. Fig. 19 shows the comparison of the charge sharing scan in the non-irradiated area with the two highly irradiated areas with opposite $V_{f d}$ gradient. The three sets of data look similar with no evidence of an asymmetry leading to the conclusion that the effects of the transverse 
electric fields produce distortions of less than 2 microns in the reconstructed cluster position for fluence gradients of $\sim 4 \times 10^{14} \mathrm{p} / \mathrm{cm}^{3}$. This is approximately the spatial resolution expected from this procedure. More precise measurements are planned to reduce this uncertainty.

The strips \#760-761 have been studied as a function of radial position in the outer radial section. Fig. 18, 20 and 21 show the $\eta$ scan in the innermost, intermediate and outer part of the strip, where the pitch is $78 \mu \mathrm{m}, 95 \mu \mathrm{m}$ and $118 \mu \mathrm{m}$ respectively. The irradiation was not collinear with the strips and there is a gradient along the strips as well as across them. Fig. 22 shows the CCE curves measured in the three positions, clearly indicating a difference in $V_{f d}$. This explains the difference in the different bias voltage behaviour of the $\eta$ scan for the different regions. In Fig. 20 and 21 the detector is non-depleted at 150 and 200 volts (positions 1 and 2 of Fig. 22) and the data are well separated from the data at 400 volts, when the detector is fully depleted. In Fig. 18 the detector is depleted at 200 volts (Pos. 3 of Fig. 22) and the set of data taken at 200 and 400 volts of applied bias almost superimpose each other.

In the $\eta$ scan the solid line represents the curve for ideal resolution. It is apparent that the charge sharing between neighbour strips in the irradiated part of the detector is much closer to the ideal resolution when the detector is biased below depletion. The presence of a non-depleted layer next to the read out strips enhances charge division, as it is shown in Fig. 23, where the laser was focused between strips $\# 740-741$, close to the \#741. A significant fraction of the charge is distributed over 4 strips at low bias voltages and decreases significantly by increasing the bias. A similar situation is not found in non-irradiated detectors, where the charge division for bias voltages below and above full depletion is more similar (Fig. 14).

Data were taken in a region with wide inter-strip pitch and intermediate routing lines. The presence of the $17 \mu \mathrm{m}$ wide routing line prevented the accurate study of the middle of the inter-strip space. A further study to be carried out in the outermost part of the inner sector (strip pitch $\sim 50 \mu \mathrm{m}$ and no routing line) will give a more precise measurement of the $\eta$ function.

\section{d. Effect of the routing-line on the collected charge}

The non-uniform irradiation permits the study of the effect of the routing lines on the CCE as a function of the dose. After the type inversion of the detector bulk from n-type to p-type because of the radiation damage, in the highly irradiated regions the junction side migrates from the back plane of the detector towards the readout strips. When the detector is under-depleted a low electric field region separates the active volume from the read out strips (double junction effects [11] are of lesser importance and are not addressed here). When the detector has not inverted, the p-n junction, and therefore the high field region, is on the side of the implanted p-type strips.

The electric field distribution influences the charge trapping as well as the signal shape and duration. In the case of the particular geometry of the LHCb Phi detector, a small signal could be induced on the routing lines in the outer segment (Fig. 5), which is dependent on the electric field distribution. The fraction of the signal measured on the routing lines as a function of the irradiation dose and of the bias applied to the detector has been studied.

In the non-irradiated region of the detector no charge was observed on the routing line at any bias voltage. Some loss of the charge to the routing has been observed in the irradiated and type inverted region, as shown in Fig. 24. The highest 
charge observed on the routing line coincides with the highest fluence region. In general, the charge in the routing line decreases strongly with bias, and becomes negligible for bias voltages above $V_{f d}$.

The ratio of the width of the routing line to the width of the read-out strips can influence the amount of the charge collected by the routing lines, because of the high relative capacitance for high ratios. The width of the routing line is constant $(17 \mu \mathrm{m})$ while the width of the outer strips increases with radius, as described in section II. Fig. 25 shows the ratio of the charge collected by the routing line to the total charge for five radial positions from the inner to the outer part of the detector. The strip width, as measured using the deficit of signal caused by the screening to the laser light from the metal read-out strip was $16,20,25,30,35 \mu \mathrm{m}$ for position 1 to 5 respectively. The effect of the lower depletion voltage in the outer part of the strip is clearly shown by the earlier decrease of the signal on the routing line for the outer positions. The effect of the relative width of the routing line to the strip, which varies from $\sim 1$ to $\sim 0.5$ going outwards, is not evident. (This would appear as a trend in the maximum sharing observed as a function of voltage.)

\section{Conclusions}

The infrared laser set-up is an effective tool for studying the effects of inhomogeneous irradiation. The measurement of the charge collection properties across the irradiated LHCb-Phi detector allowed the reconstruction of the measured irradiation profile.

The study of the charge sharing between neighbouring strips as a function of the local position shows no evidence of distortion of the resolution as a consequence of a distorted electric field resulting from inhomogeneous irradiation.

Evidence has been found of increased charge sharing in the irradiated and type inverted part of the detector when biased below $V_{f d}$. This effect is expected because of the presence of the non-depleted bulk next to the read-out strip. The enhanced resolution implied by the increased charge sharing is not necessarily beneficial. Operating ( $p$-in-n) sensors under-depleted implies a large reduction in charge collection efficiency. Post-irradiation it is important to operate the detector with high charge collection to optimise the signal to noise ratio. An alternate choice of the diode structure, like n-strips (n-in-n) or n-strips in p-bulk, would greatly benefit the operation of irradiated detectors [12-14].

The measured shape of the noise at high bias voltages on the irradiated sector of the detector (where the $V_{f d}$ varies from $50 \mathrm{~V}$ to $280 \mathrm{~V}$ ) is compatible with noise induced by micro-discharges. The micro-discharge effect depends on the strength of the electric field and not on the applied bias. With the same applied bias voltage the electric field is higher for lower $V_{f d}$. In this region the noise is inversely proportional to $V_{f d}$ confirming the hypothesis on the origin of the noise.

The unique geometry of the LHCb-phi detectors includes metal lines to route the innermost strips to the bonding pads located on the outer end of the detector. The metal lines run symmetrically between the outer strips. The effect of these metal lines on the charge collection has been studied. When the detector is type inverted and biased below depletion, a fraction of the total charge is seen by the routing line and therefore lost. This effect is suppressed when the detector is depleted. Moreover, this effect is not seen in the non-irradiated part, suggesting that it is correlated with the presence of the non-depleted layer next to the strips. In case of read-out from the junction side, as for n-in-n or n-in-p detectors, no charge loss in the routing lines is 
predicted, improving the performances for non-depleted operation, as compared with p-in-n detectors.

\section{References}

[1] C. Parkes and T. Bowcock, Nucl. Instr. And Meth. In Phys. Research A435 (1999)65-73.

[2] LHCb Technical Proposal, CERN/LHCC98-4.

[3] Micron Semiconductors, 1 Royal Buildings, Marlborough Road, Lancing, Sussex, BN15 8UN (UK).

[4] M. Glaser et al., Nucl. Instr. And Meth. In Phys. Research A426 (1999)72-77. See also http://irradiation.web.cern.ch/irradiation/.

[5] E. Leòn-Floriàn et. Al. "Particle fluence measurements by activation technique for radiation damage", CERN-ECP/95-15.

[6] A. Ruzin et al., Nucl. Instr. And Meth. In Phys. Research A,426 (1999)94-98.

[7] J. Kaplon et al. IEEE Transact. On Nucl. Sci. 44 (1997)2 98.

[8] G. Casse et al., Nucl. Instr. And Meth. In Phys. Research A 438 (1999) 429-432.

[9] S. Martí I García et al. "A model of charge collection for irradiated $p+n$ detectors", presented at Vertex 2000, 9th International Workshop on Vertex Detectors, Sleeping Bear Dunes National Lakeshore - Michigan, USA, to be published in NIMA.

[10] T. Oshugi et al., Nucl. Instr. And Meth. In Phys. Research A, 342 (1994) 22.

[11] G. Casse et al., Nucl. Instr. And Meth. In Phys. Research A 426 (1999) 140.

[12] S. Martí I García et al., Nucl. Instr. And Meth. In Phys. Research A426 (1999)24 27.

[13] P. Allport et al., Nucl. Instr. And Meth. In Phys. Research A435 (1999) 74-79.

[14] T. Bowcock, Silicon Technology for the LHCb Vertex Detector, LHCb/98-048 


\section{Figures}

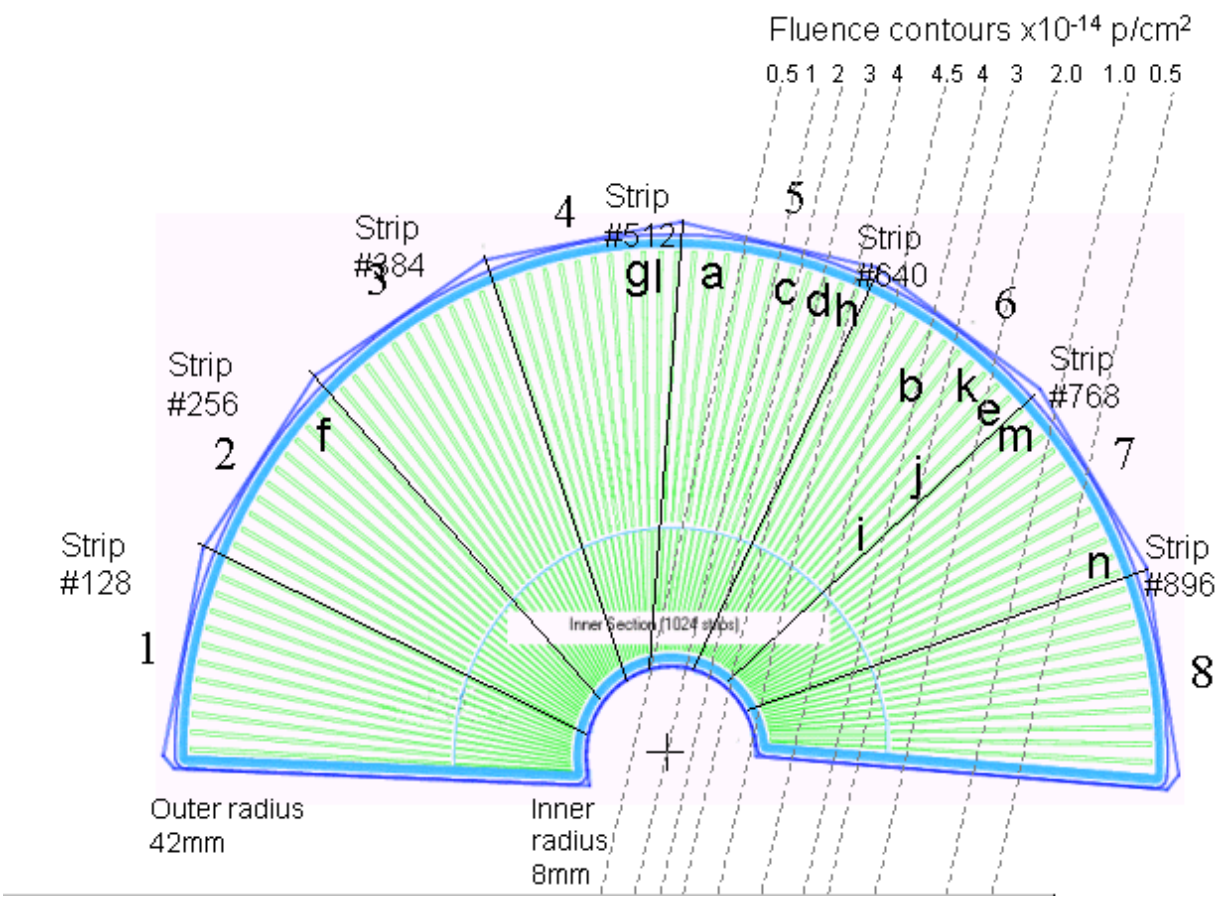

Fig. 1: Schematic draft of the LHCb-Phi detector. Diagram contains approximate fluence contours for the irradiated detector and some indicative measuring positions labelled $(a-n)$, most of which are along the outer radius of the sensor.

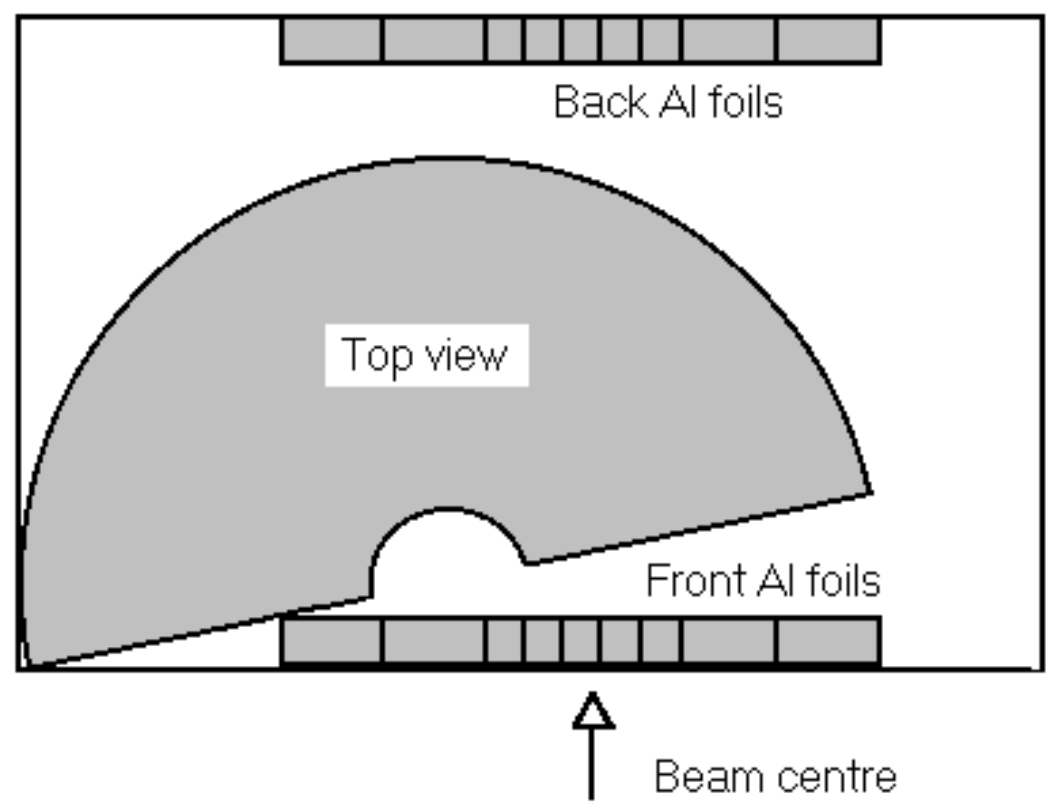

Fig. 2: Diagram of the inhomogeneous irradiation set-up. 


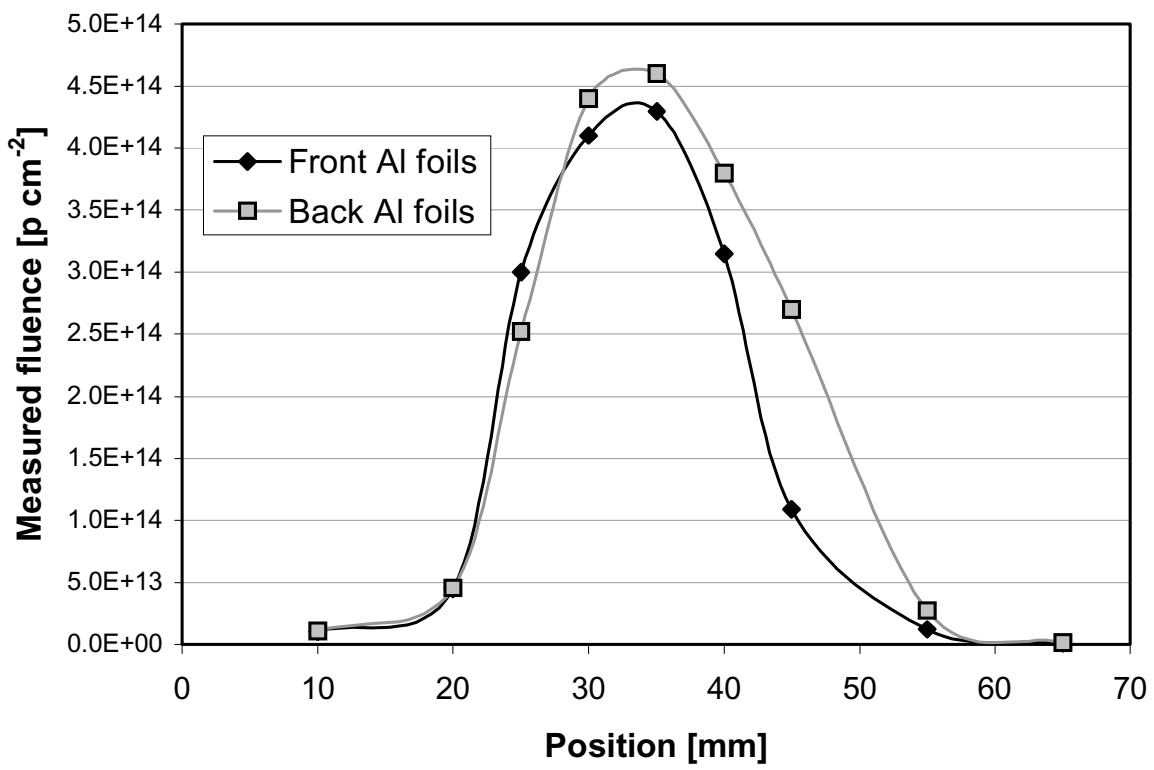

Fig. 3 Beam profile as measured with the Al foils activation method on the before and after the beam crossing the detector.

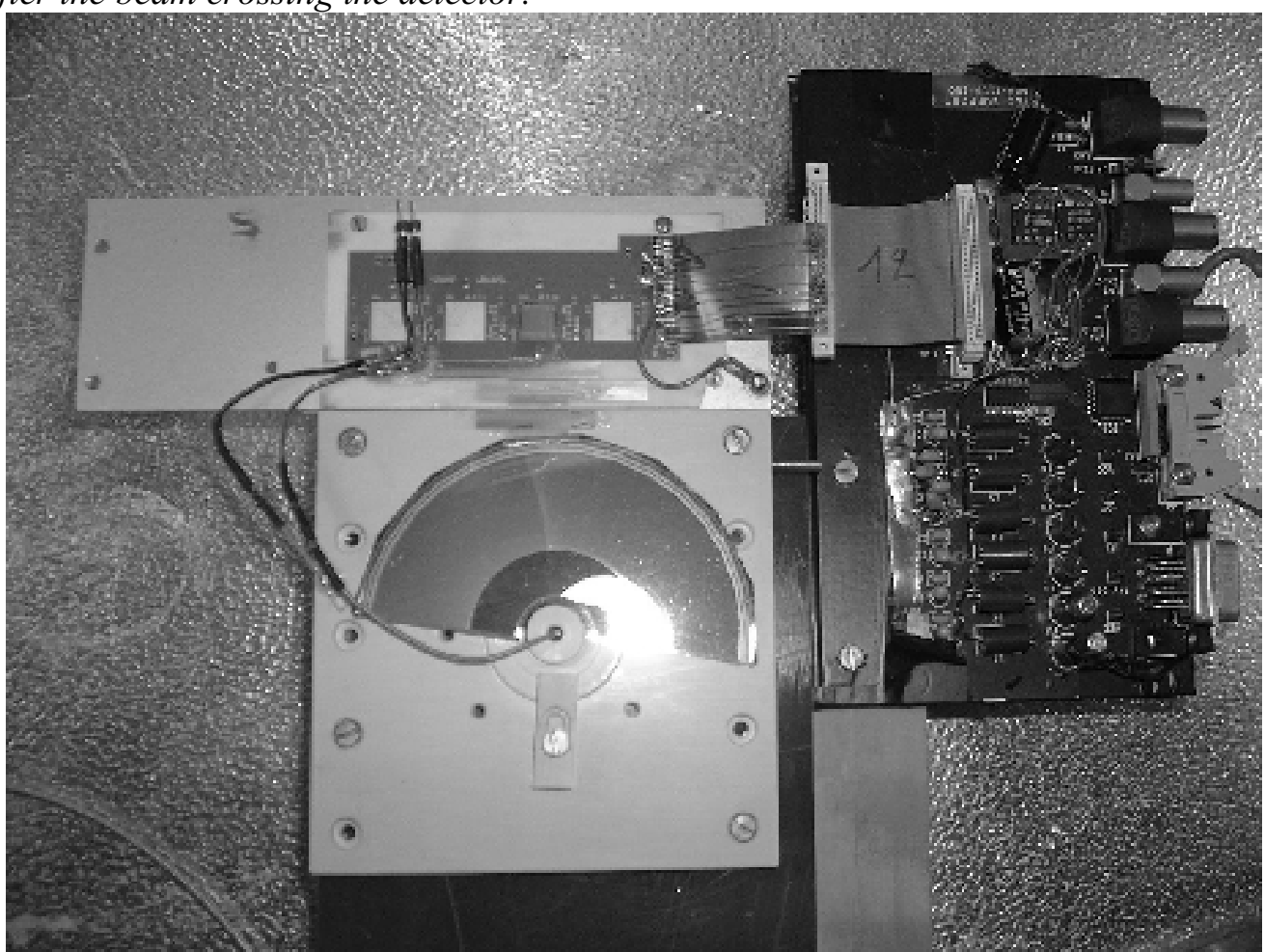

Fig. 4 Rebondable support for the detector. The support can pivot around the centre to allow successive read-out of the various sectors. 


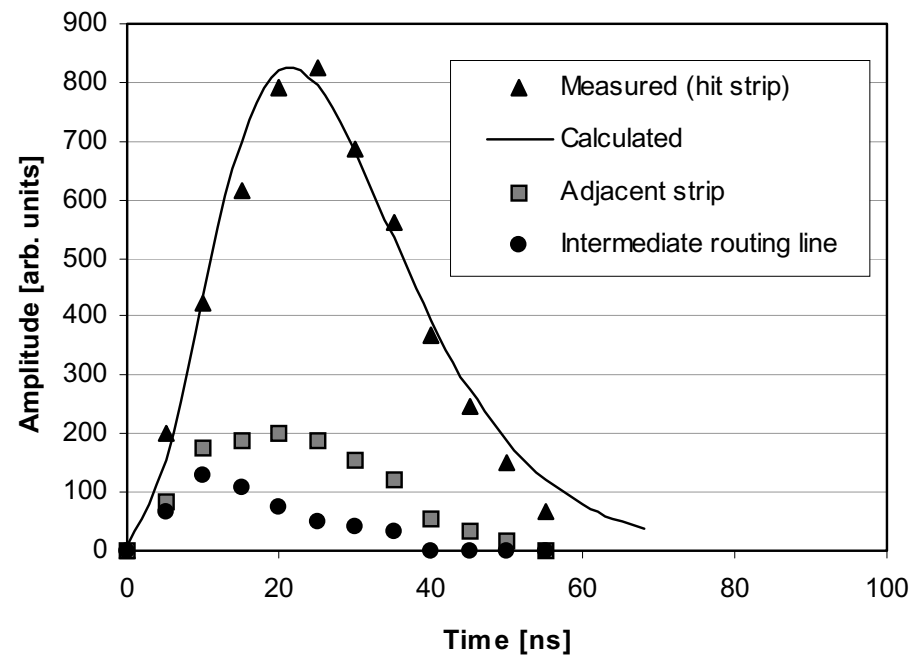

Fig. 5 Time scan to reconstruct the pulse shape of the SCT128 chip. The solid line is the predicted SCT128a pulse shape. In the same plot are shown the hit strip, a neighbour one and the intermediate routing line.

6-Mar-日1

$17: 15: 27$

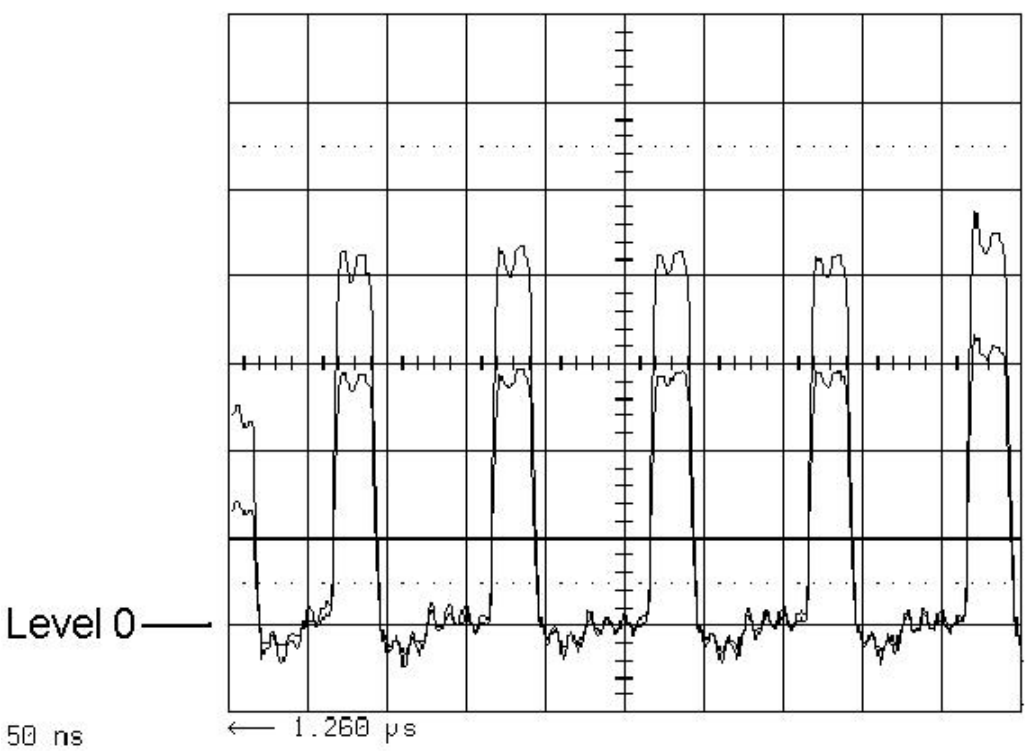

Fig. 6: Calibration signal from the SCT128 chip. Two different heights of the internal calibration (a charge is injected every fourth channel) have been used to show the overshoot in the time bin that follows a hit one. 


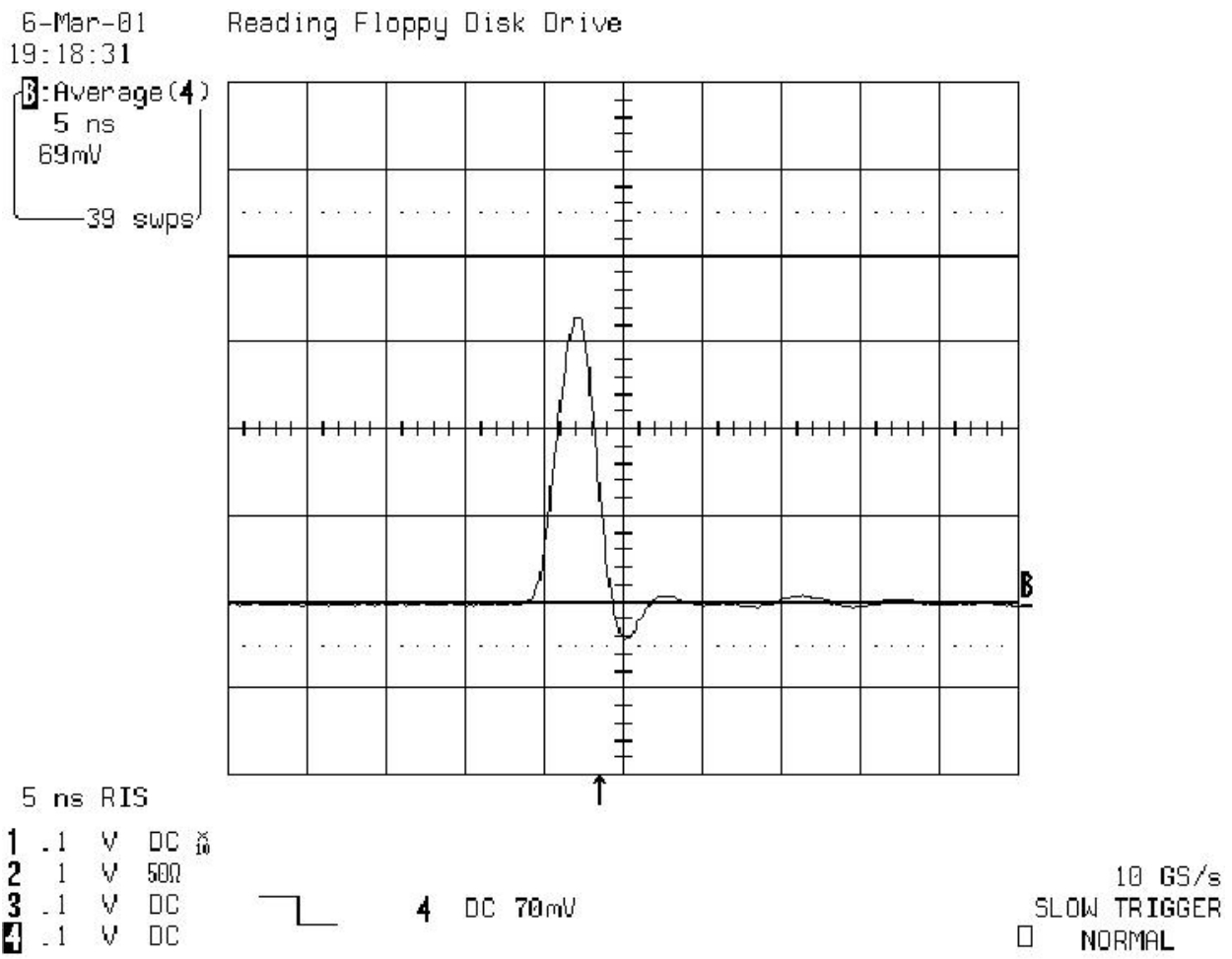

Fig. 7: Infrared (1060 $\mathrm{nm}$ wavelength) laser pulse as measured by a fast optical/electrical converter.

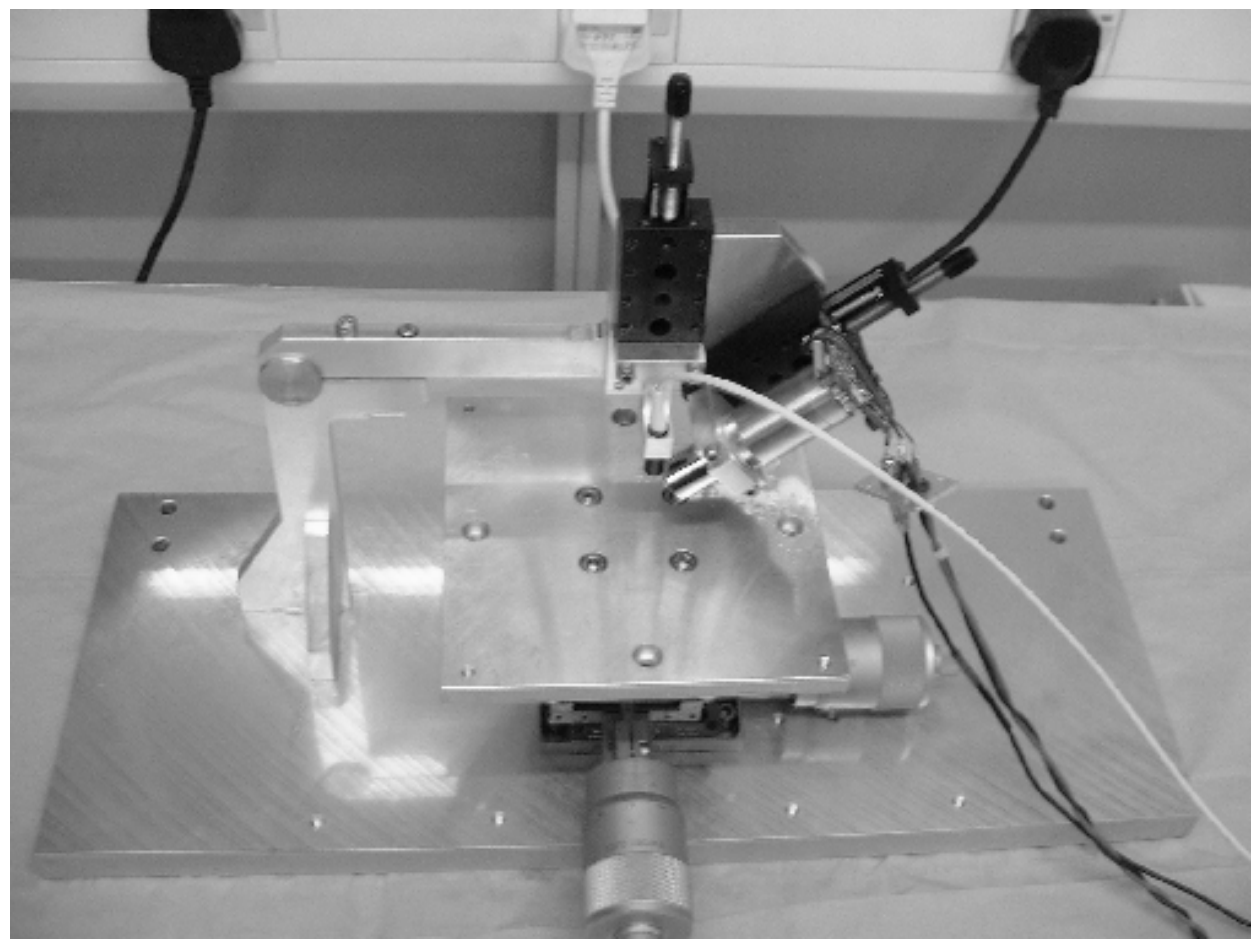

Fig. 8: $x$-y table with micromanipulator, laser focuser and CCD camera. 


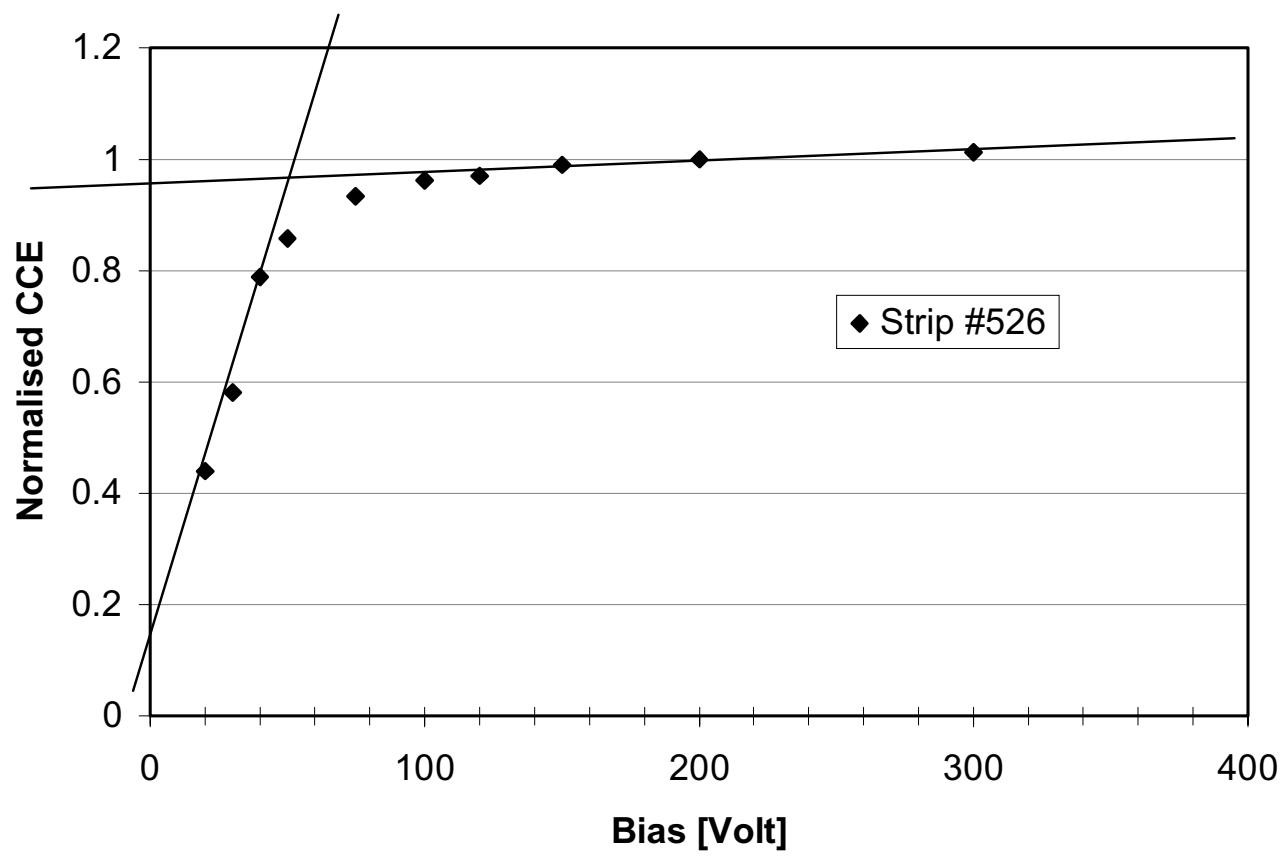

Fig. 9(a) Example of evaluation of $V_{\text {fd }}$ from the charge collection curve as a function of bias. In this low fluence case $V_{f d}=53 \mathrm{~V} . V_{f d}$ is defined to be the intersection of the two lines. Strip \#526 was measured at position a (see Figure 1).

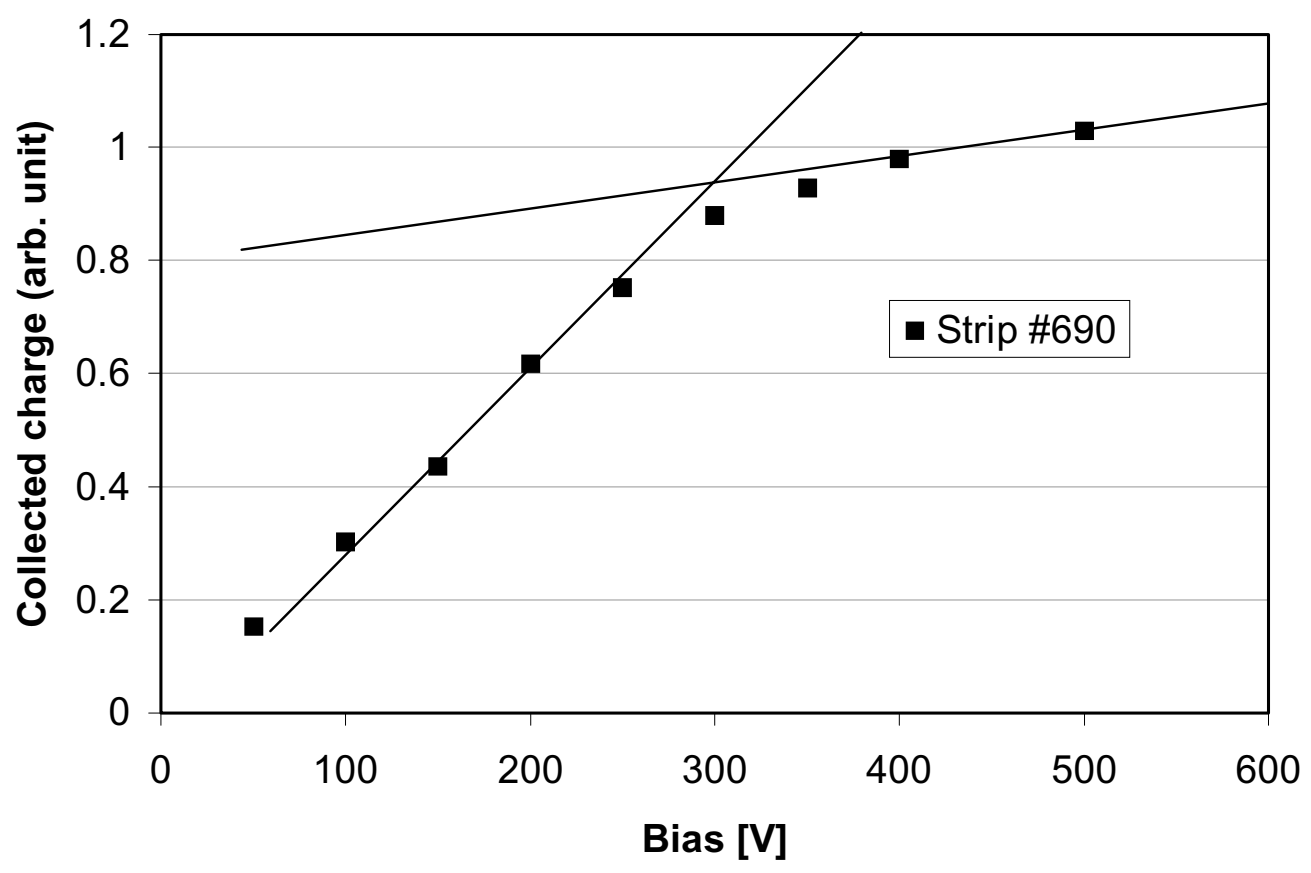

Fig. 9(b): Example of evaluation of $V_{f d}$ from the charge collection curve as a function of bias. In this high fluence case $V_{f d}=300 \mathrm{~V} . V_{f d}$ is defined to be the intersection of the two lines. Strip \#690 was measured at position b (see Figure 1). 


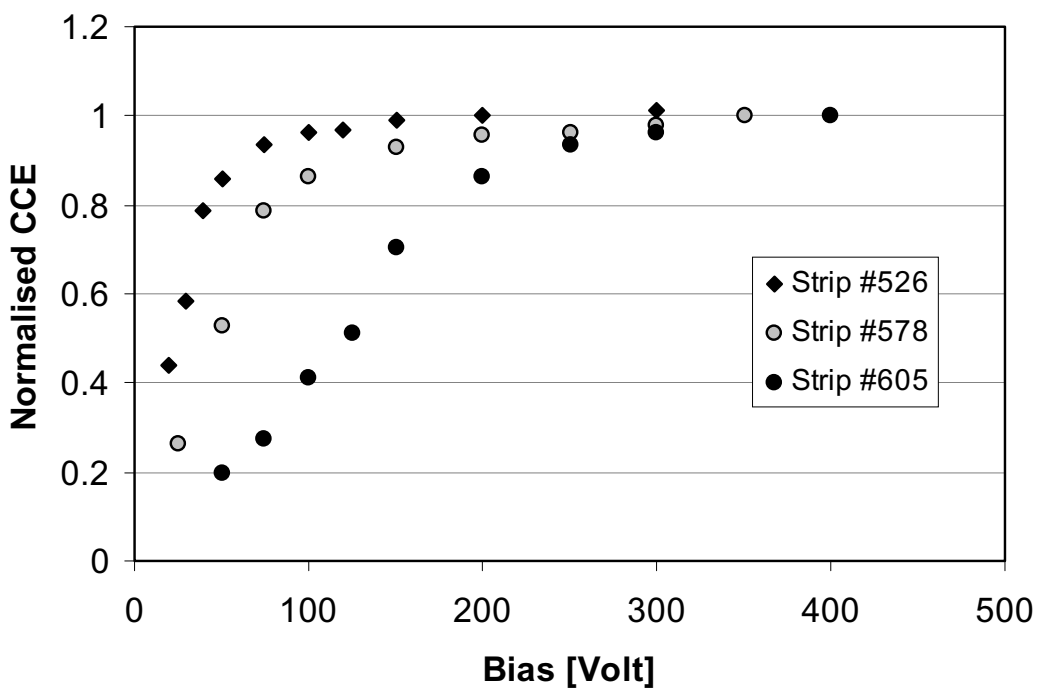

Fig. 10: Sample of CCE curves in three different locations of the detector with different irradiation fluences. Strip \#526 is in the lowest region, strip \#578 in an intermediated region and strip \#605 in a region of high flux. The degradation in depletion voltage can be clearly seen. Strips \#526, 578 and 605 were measured at positions a,c and d respectively (see Figure 1).

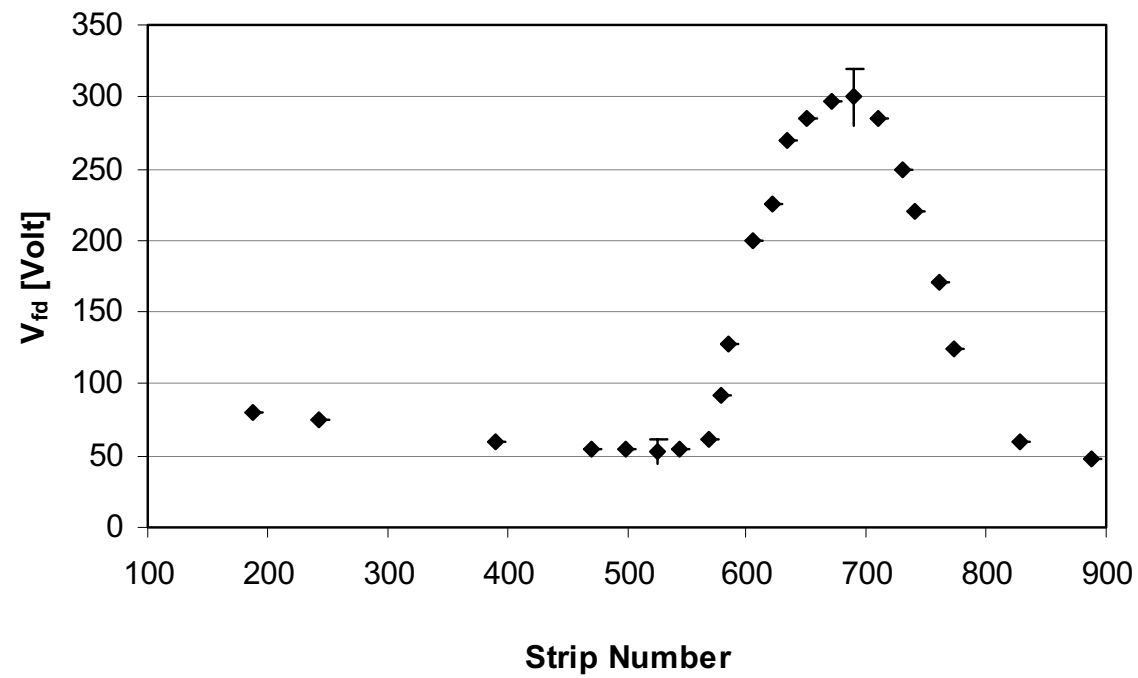

Fig. 11: Full depletion voltage profile across the detector, as evaluated from the CCE curves measured on the outer end of the outer strips. Two typical error bars are shown for the low and high $V_{f d}$ regions. The profile matches the spatial irradiation profile. (Note Channels 580 to 800 span about 25mm). 

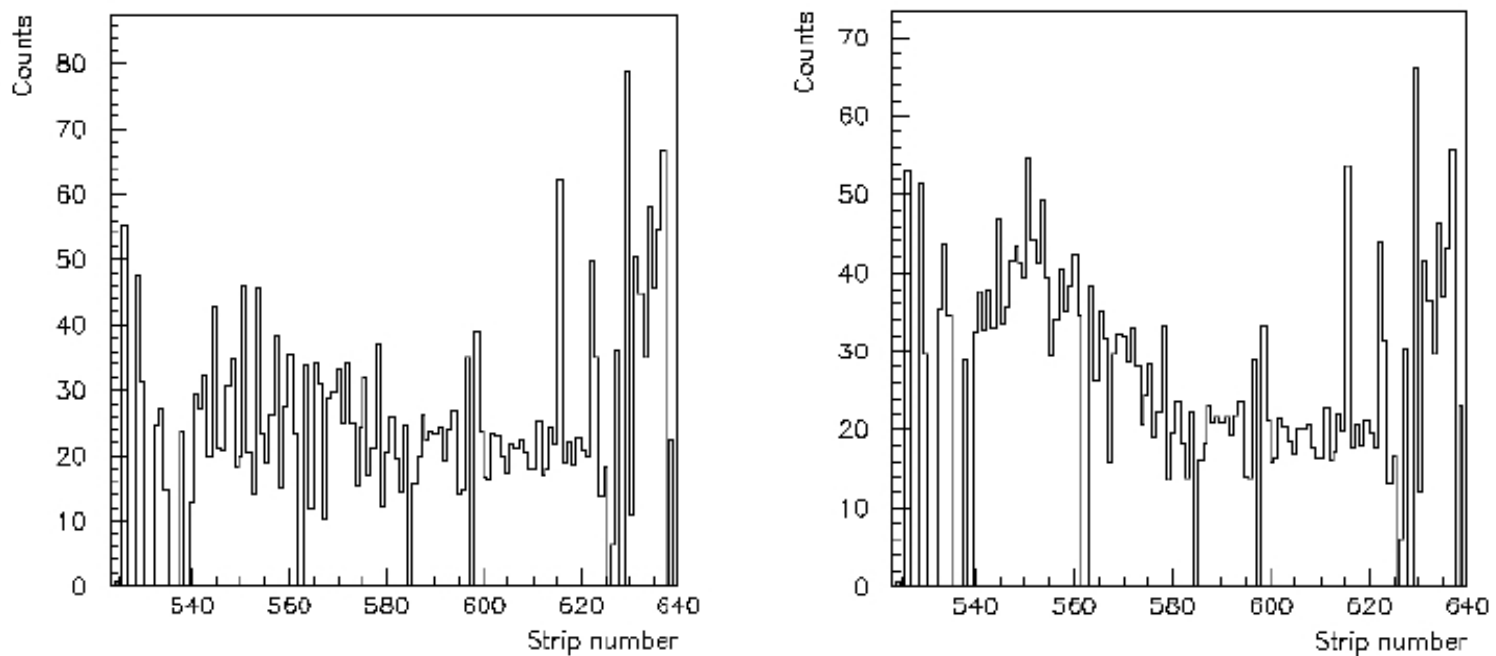

a) $V=100$ Volts

b) $V=200$ Volts
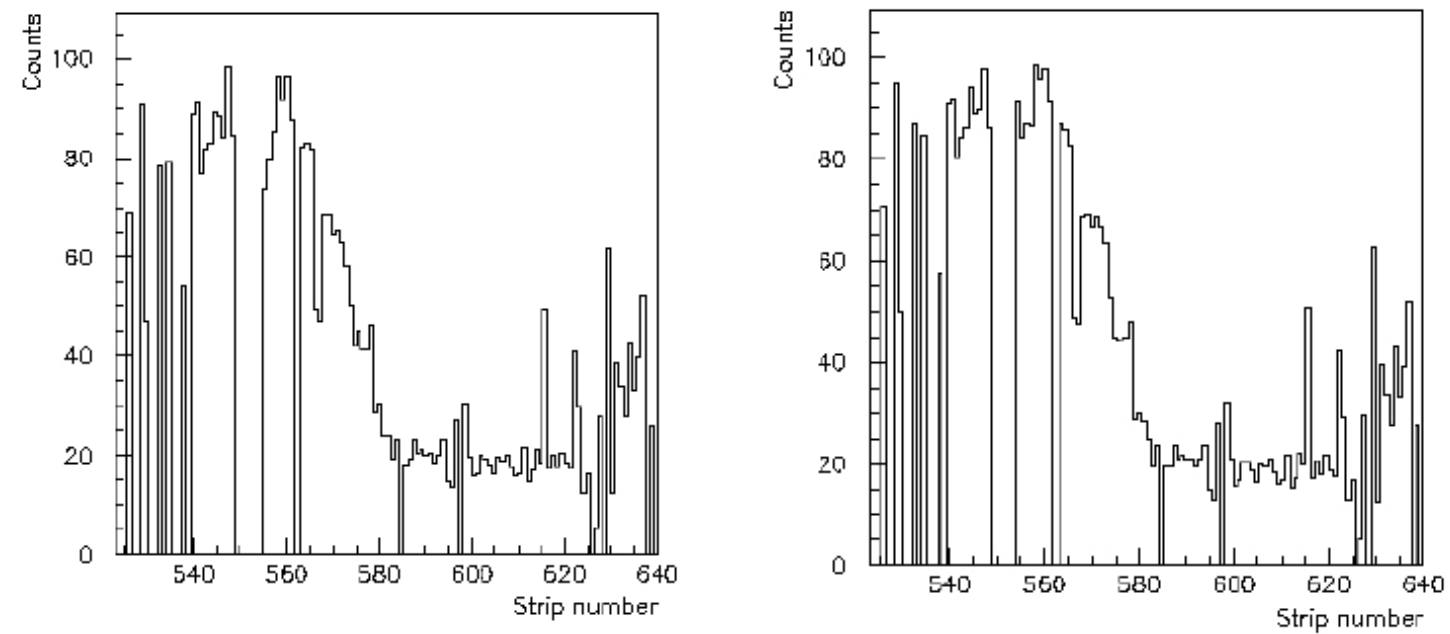

c) $V=300$ Volts

d) $V=350$ Volts

Fig. 12: Noise distribution in the sector number 5 of the irradiated detector at different bias voltages 


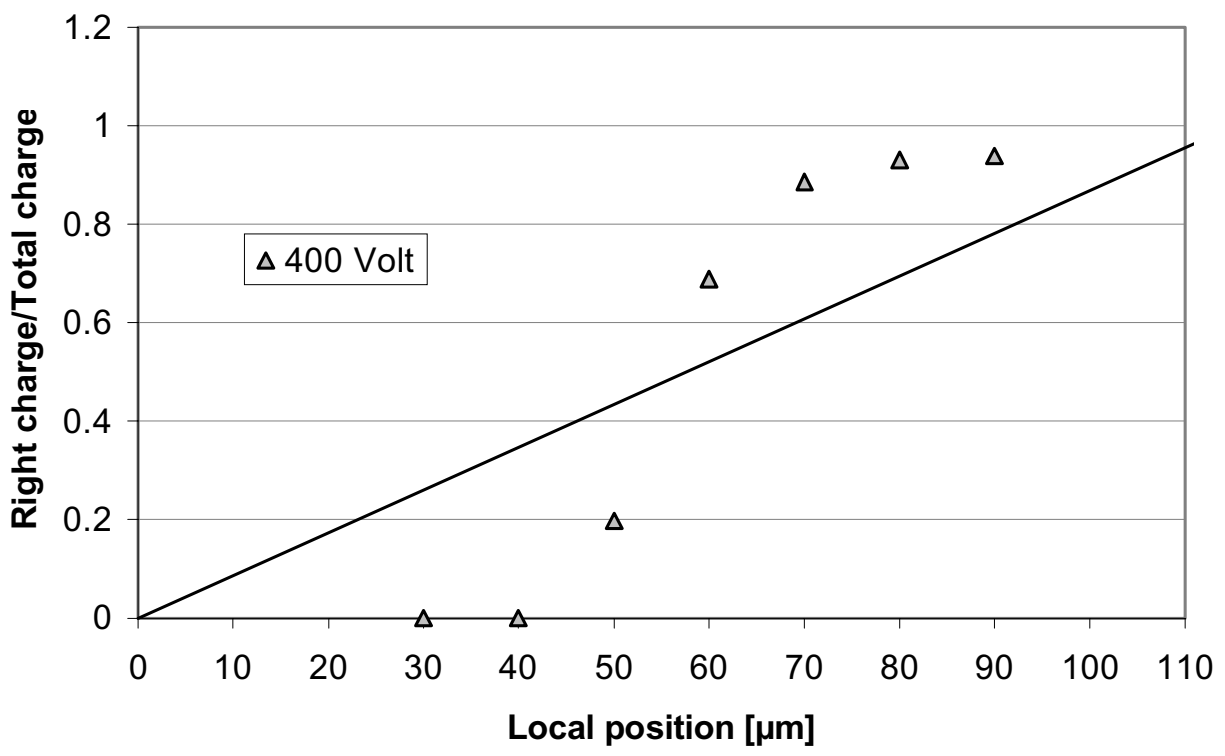

Fig. 13(a): $\eta$ scan across strip \#740 - 741. The left and right strips are connected to two consecutive electronic channels: the zero charge seen in the first two points at 400 volts is due to the overshoot (see text) and requires appropriate correction. The strips were measured at position e (see Figure 1).

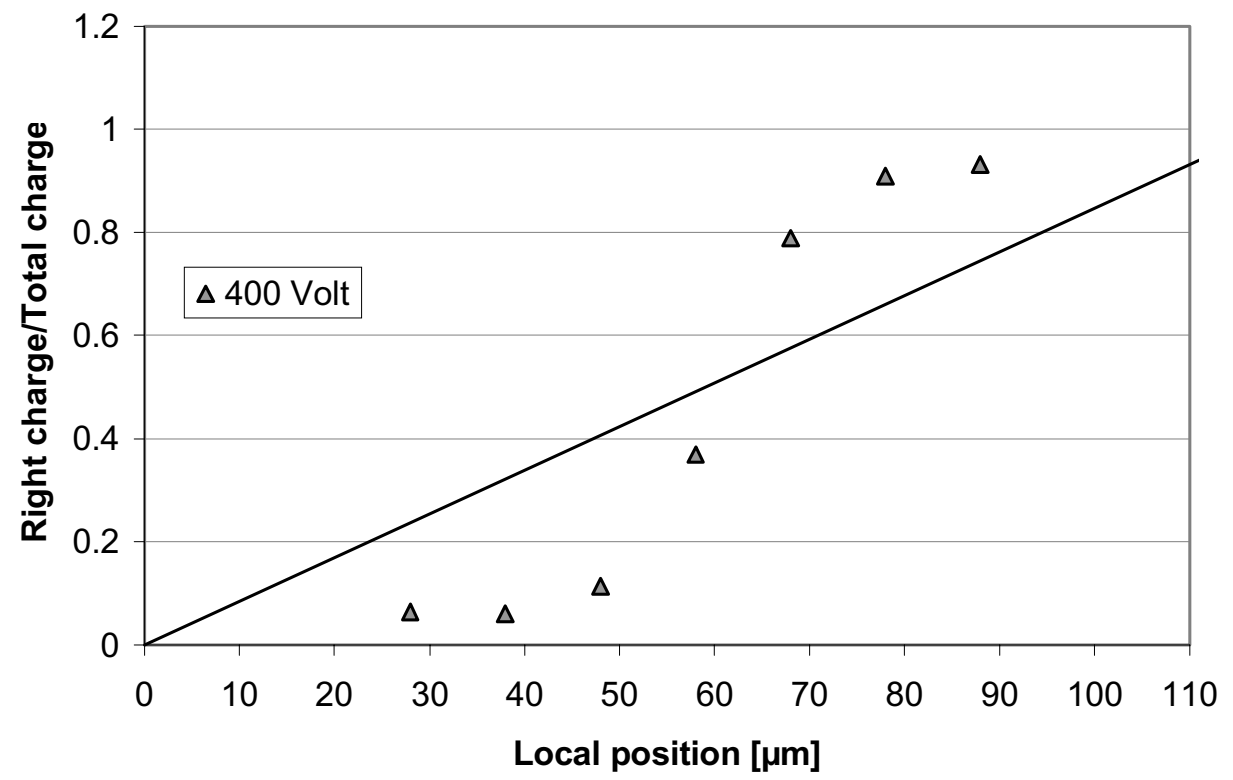

Fig. 13(b): $\eta$ scan across strip \#760 - 761 with intermediate routing lines connected to the read-out electronics, thus no correction for the overshoot in the right strip is required. 


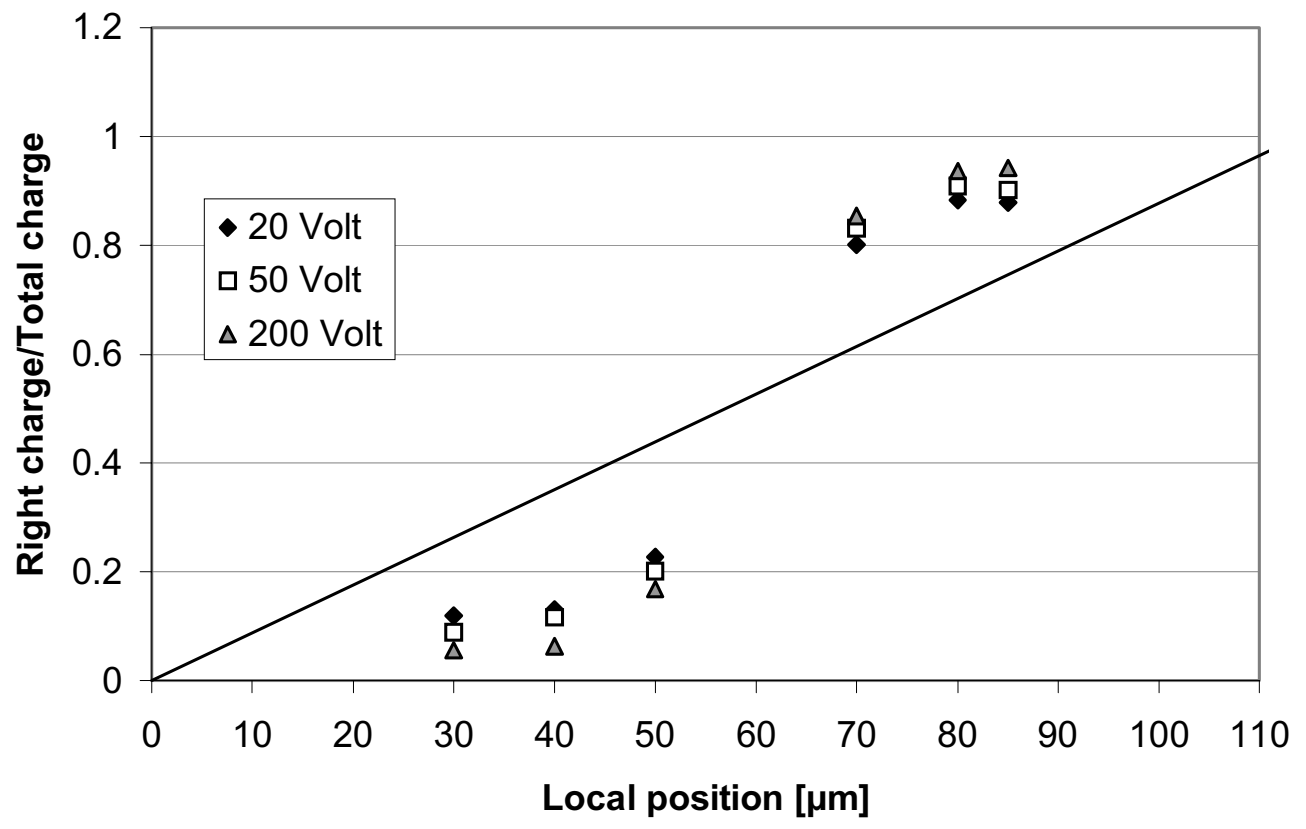

Fig. $14 \eta$ scan across strip \#244 - 245 (non irradiated region) measured at position $\boldsymbol{f}$ (see Figure 1).

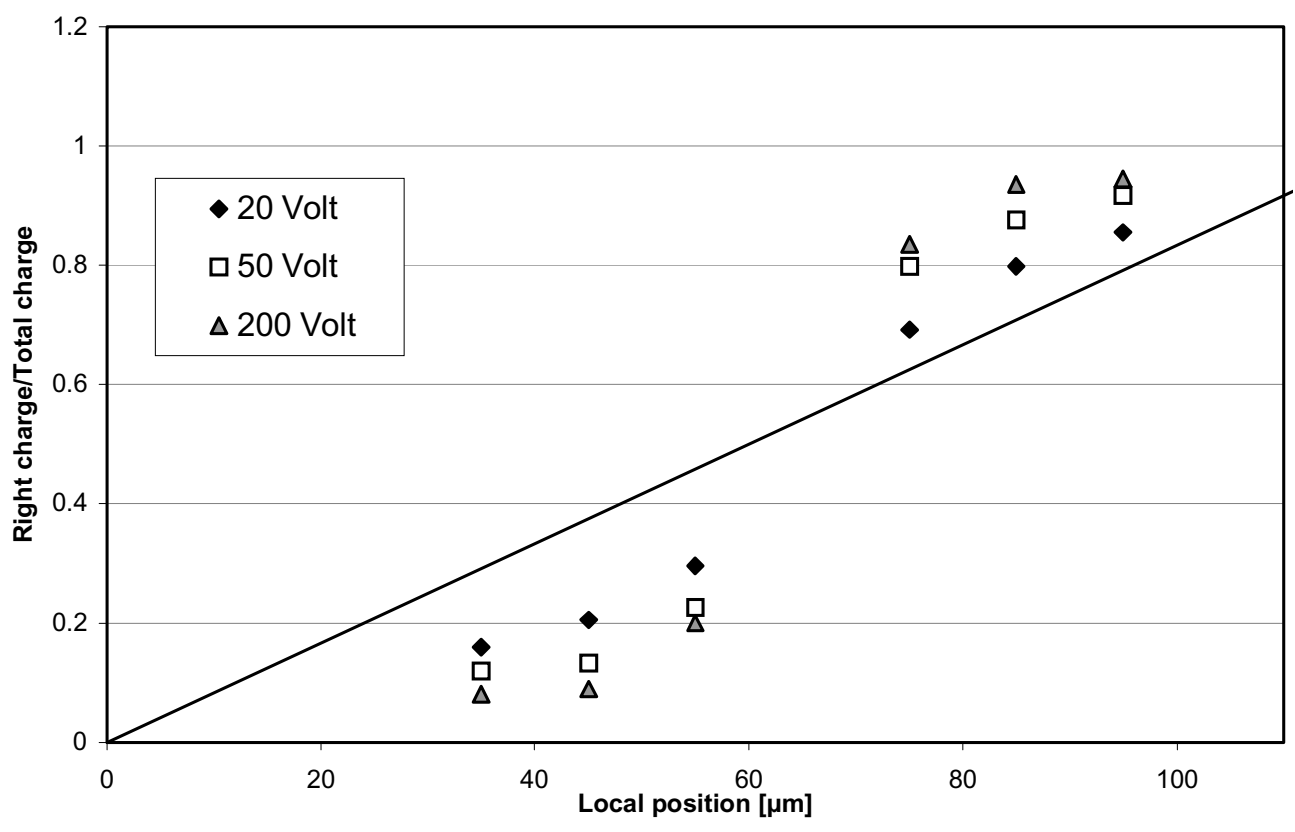

Fig. $15 \eta$ scan across strip \#490 - 491 (irradiated region with low $V_{\text {fd }}$ ) measured at position $\mathbf{g}$ (see Figure 1). 


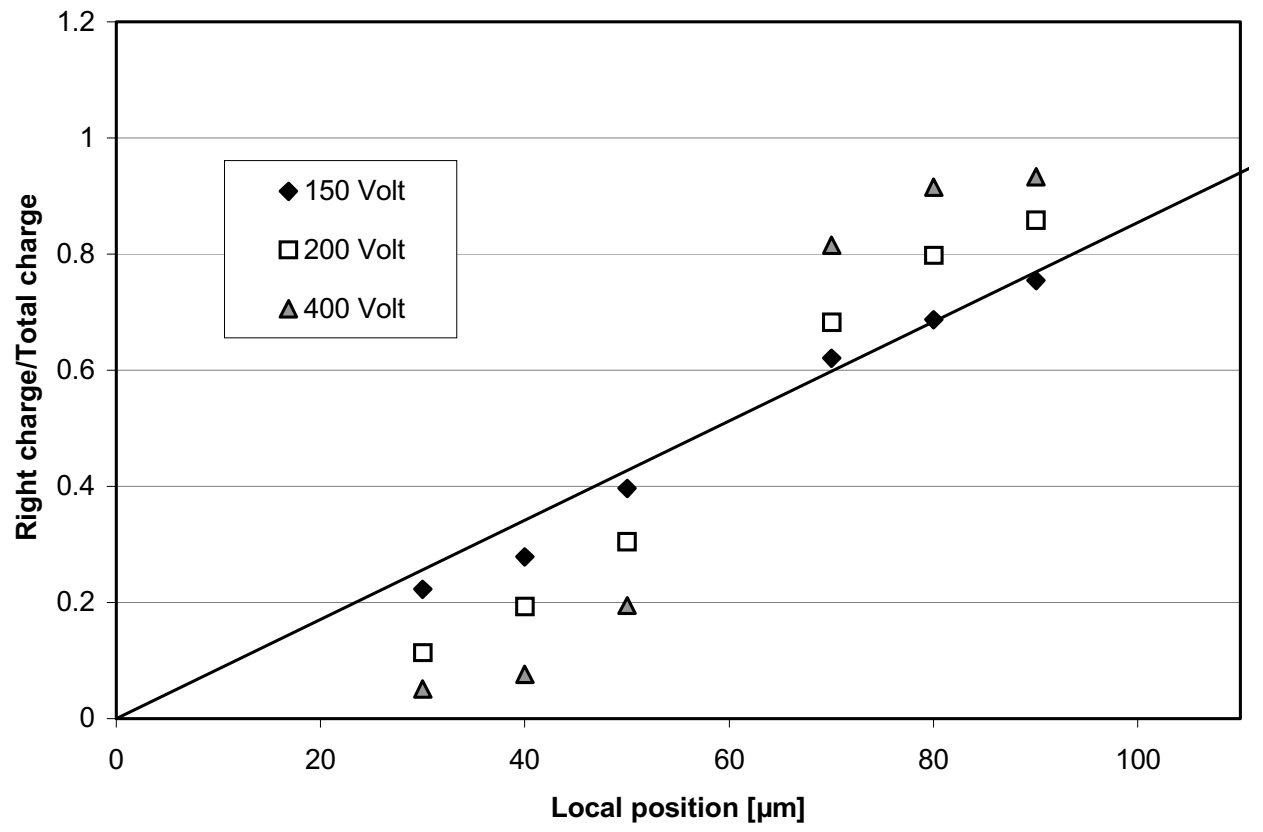

Fig. 16: $\eta$ scan across strip \#634 - 635 (irradiated region with positive gradient of $V_{f d}$ as a function of the strip number). The measuring position was $\boldsymbol{h}$ (see figure 1).

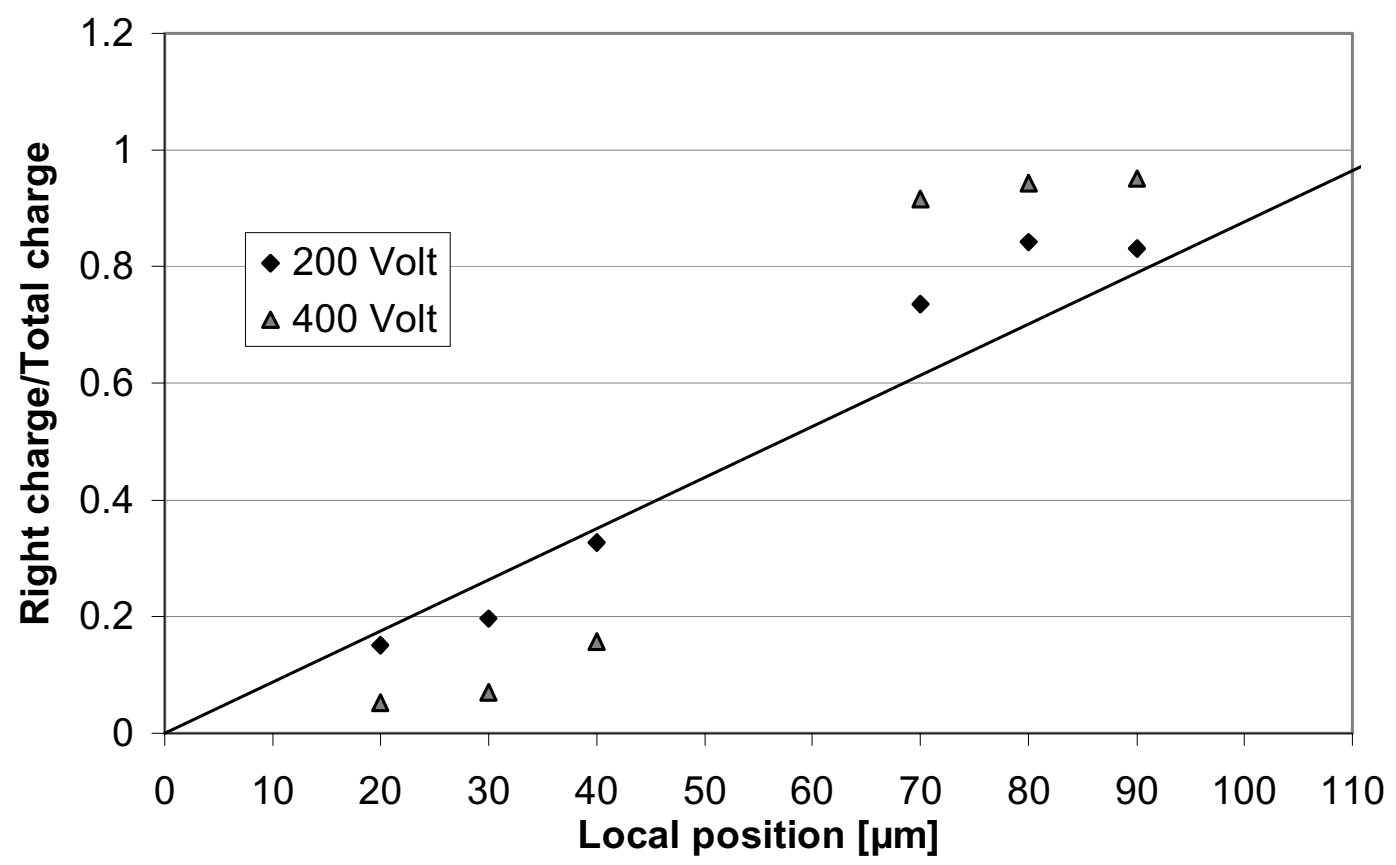

Fig. 17: $\eta$ scan across strip \#690 - 691 (heavily irradiated region). The detector was measured at position $\boldsymbol{i}$ (see figure 1). 


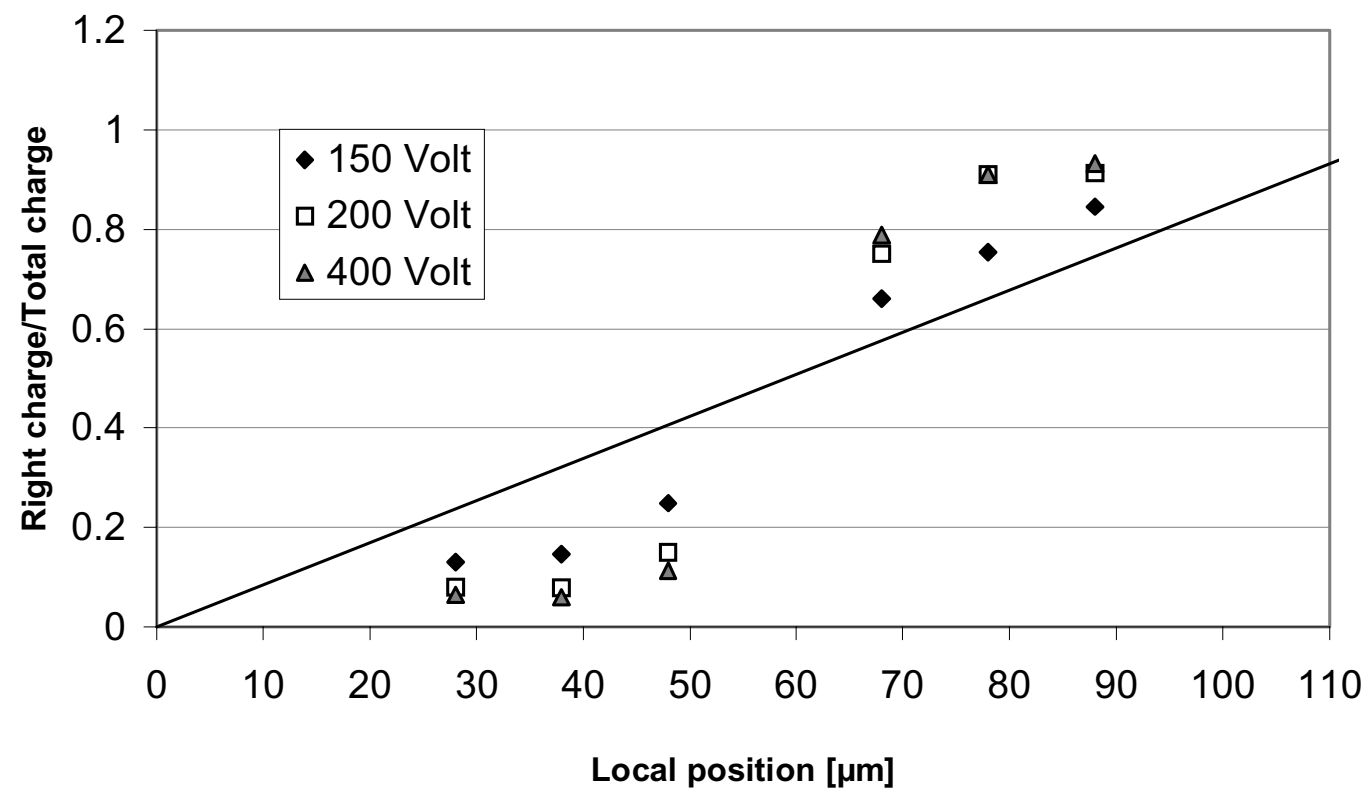

Fig. 18: $\eta$ scan across strip \#760 - 761 (irradiated region with negative gradient of $V_{f d}$ as a function of the strip number). Measured position is at $\boldsymbol{j}$ (see Figure 1).

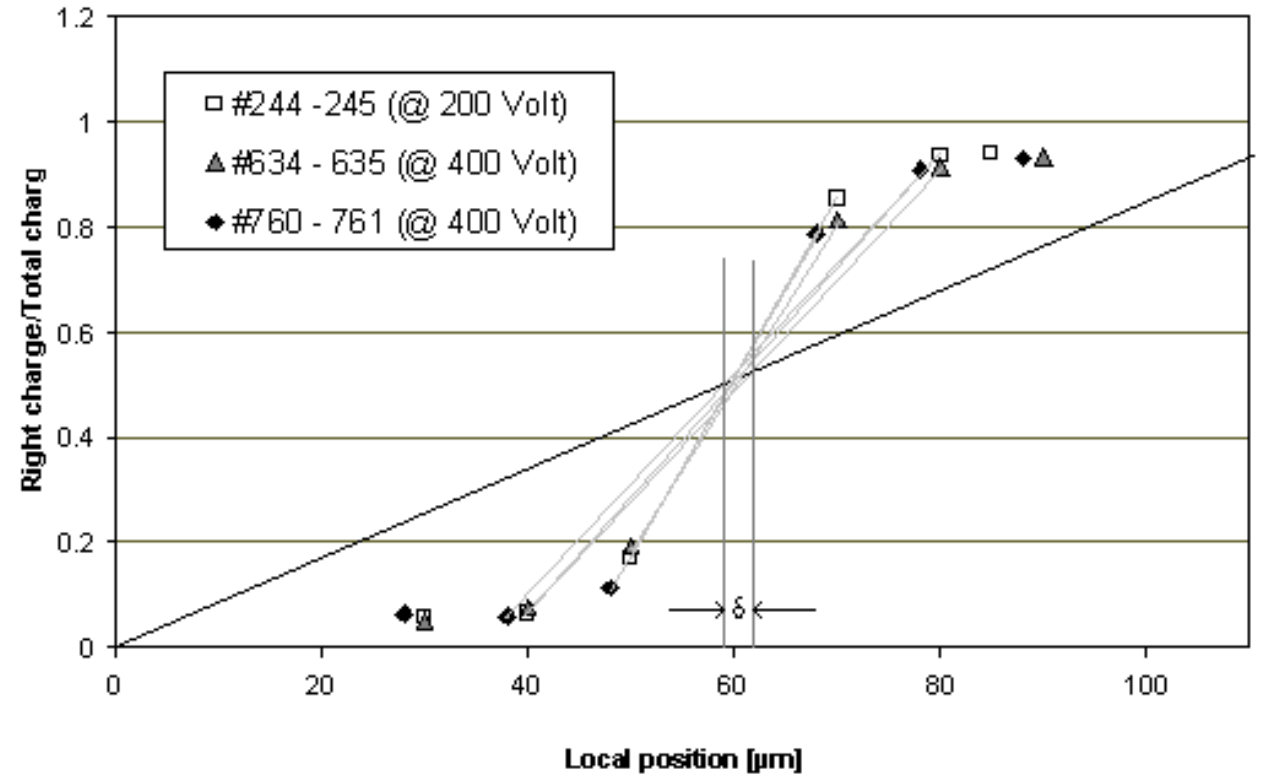

Fig. 19: Comparison of the $\eta$ scan across adjacent strips located in the non irradiated, irradiated with positive gradient of $V_{f d}$ and irradiated with negative gradient of $V_{f d}$ regions respectively. The degree to which all points from the positive and negative gradient areas on opposite the left and right hands sides of the geometric centre of the strips pass through a common point is a measure of the distortion. The spread observed $(\delta)$ is approximately \pm 2 microns. The measured strips \#244,634 and 760 correspond to measuring points $\boldsymbol{f}$, $\boldsymbol{h}$ and $\boldsymbol{e}$ (see Figure 1). 


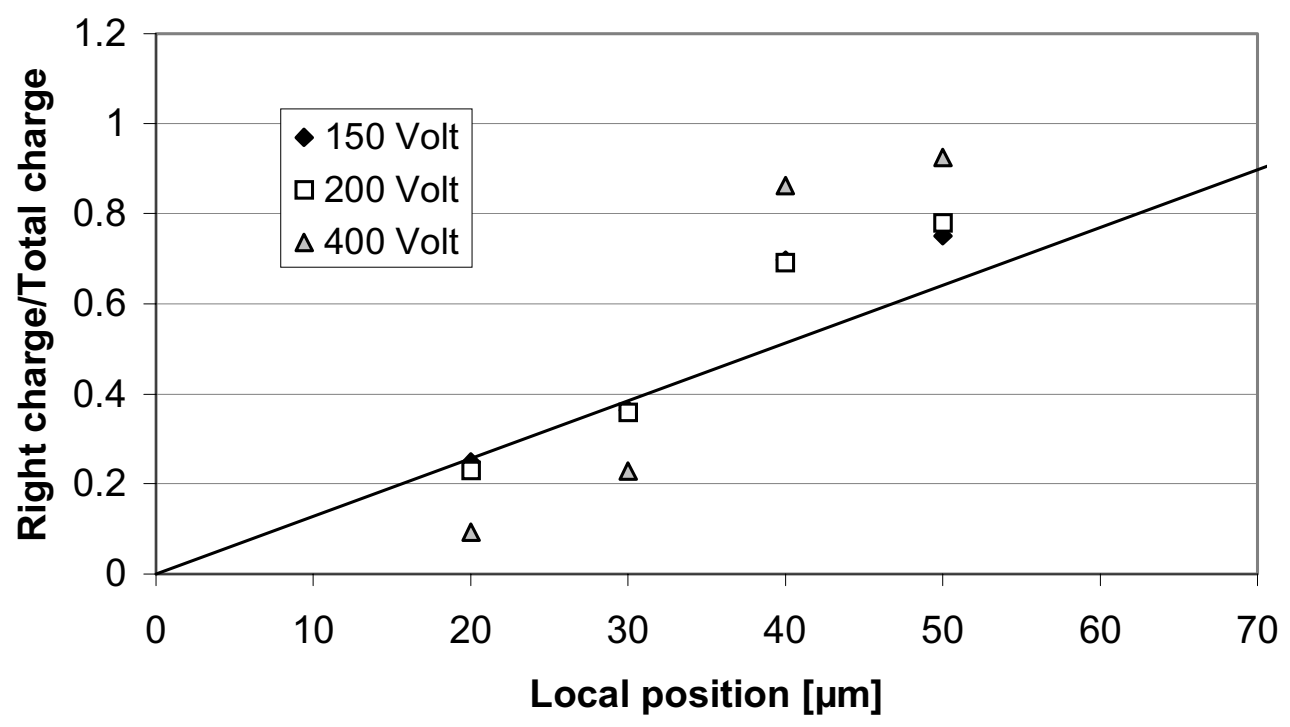

Fig. 20: $\eta$ scan across strip \#760 - 761 in the inner part (strip pitch 78 $\mu \mathrm{m})$.Measured position corresponds to $\boldsymbol{i}$ (see Figure 1)

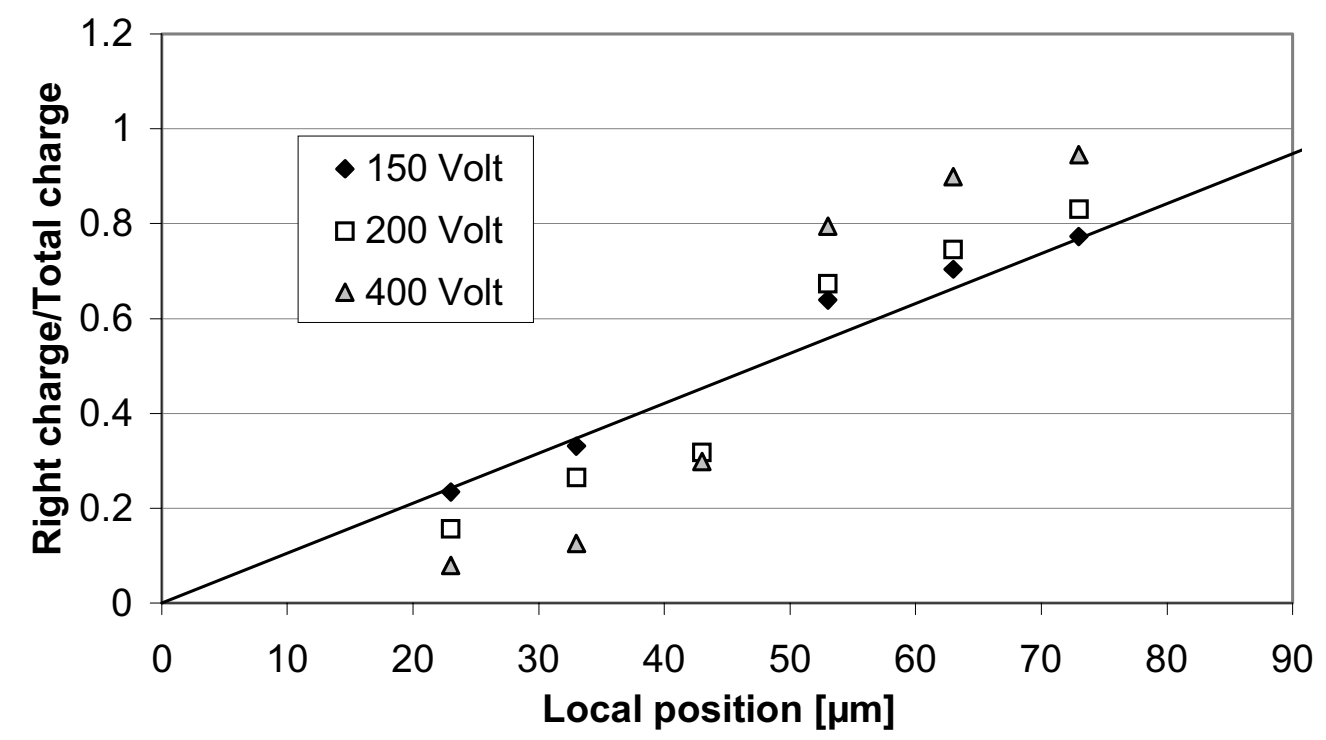

Fig. 21: $\eta$ scan across strip \#760 - 761 in the intermediate part (strip pitch $95 \mu \mathrm{m}$ and position $\boldsymbol{j}$ in Figure 1) 


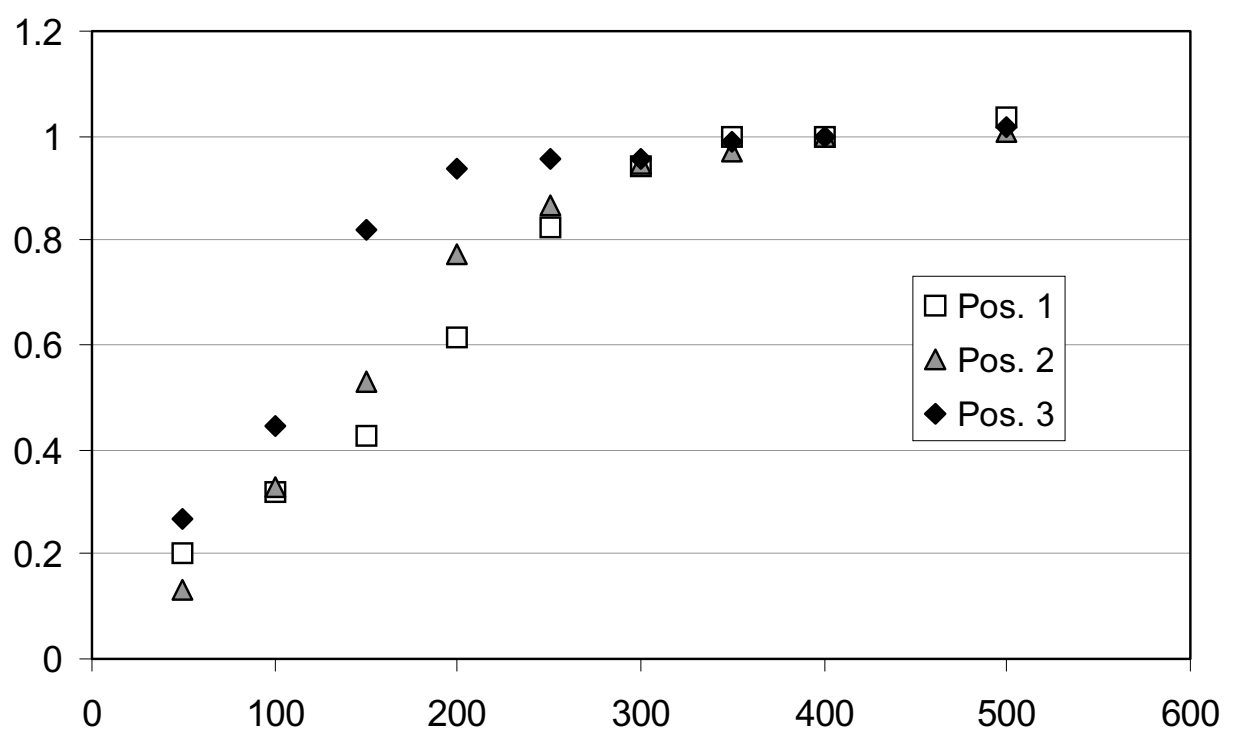

Fig. 22: CCE curves measured next to strip \#760: Pos. 1, 2 and 3 are the inner part, intermediate and outer parts of the strip (measuring points $\boldsymbol{i}, \boldsymbol{j}$, and $\boldsymbol{e}$ Figure 1).

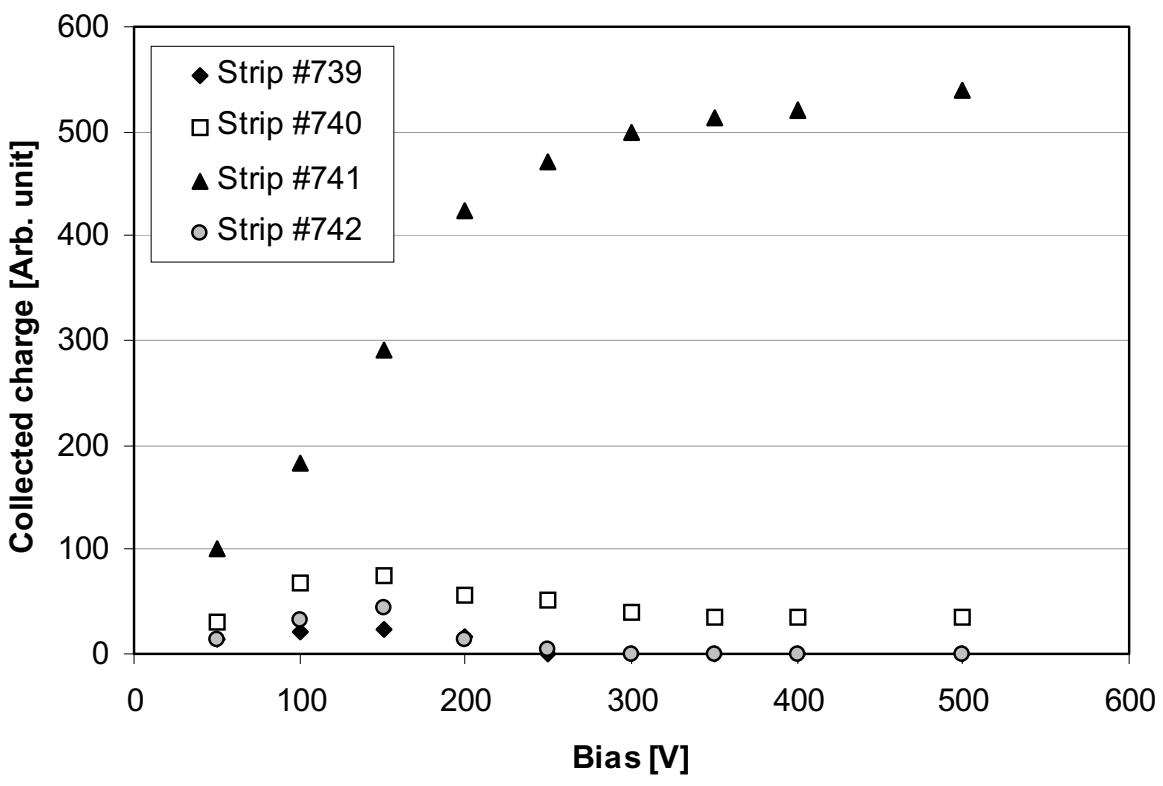

Fig. 23: Charge collection in four adjacent strips in a irradiated and type inverted region. The laser was focused between strips $\sim 740-741$, next to strip \#741. (The measuring position was $\boldsymbol{k}$, see Figure 1) 


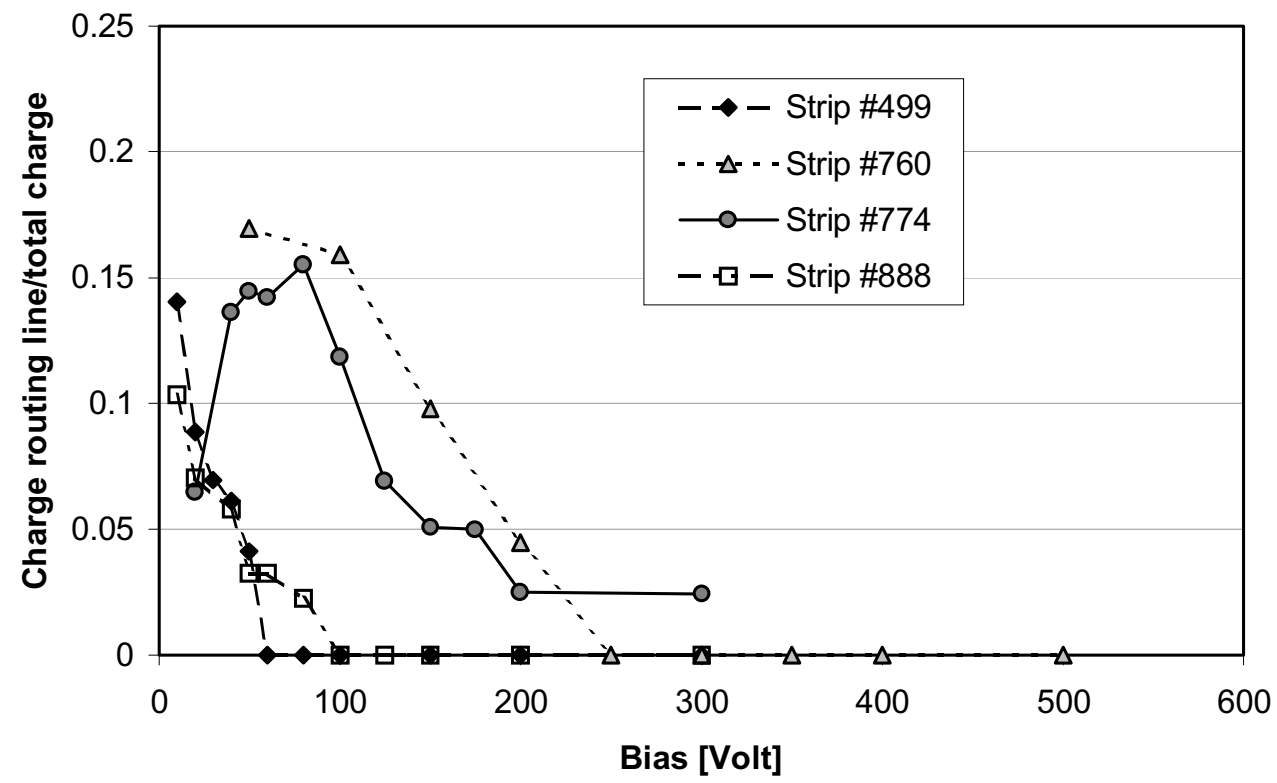

Fig. 24: Ratio between the charge collected by the routing line to the total collected charge in irradiated areas with different fluences. Strips \#499,760,774 and 888 were

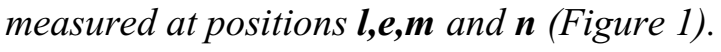

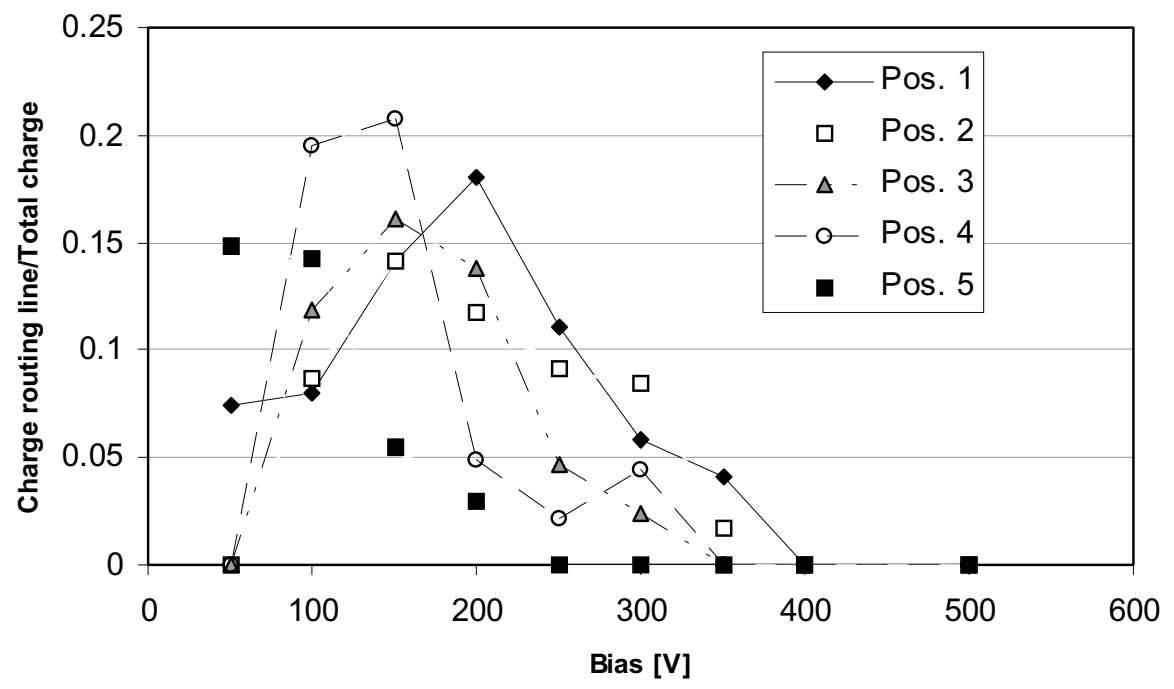

Fig. 25: Ratio between the charge collected by the routing line to the total collected charge in the proximity of strip \#760 in five equally spaced positions from the inner to the outer end of the strip. The shape of the curves reflect are dependent on the degree of depletion of the sensor at a fixed location and the geometry of the strips. The depletion effects are seen to dominate as there is no trend in the plot maximum from positions 1-5. 